OPEN ACCESS

Edited by:

Giulio Cabrini,

University of Ferrara, Italy

Reviewed by:

Onofrio Laselva,

University of Foggia, Italy

Deborah M. Cholon,

University of North Carolina at Chapel

Hill, United States

*Correspondence:

Béla Nagy Jr.

nagy.bela@med.unideb.hu

Specialty section:

This article was submitted to

Respiratory Pharmacology,

a section of the journal

Frontiers in Pharmacology

Received: 06 August 2020 Accepted: 16 April 2021

Published: 14 May 2021

Citation:

Bene Z, Fejes Z, Szanto TG, Fenyvesi $F$, Váradi J, Clarke LA, Panyi G, Macek M, Amaral MD, Balogh I and Nagy B Jr. (2021) Enhanced Expression of Human Epididymis Protein 4 (HE4) Reflecting Pro-Inflammatory Status Is Regulated by CFTR in Cystic Fibrosis Bronchial Epithelial Cells.

Front. Pharmacol. 12:592184. doi: 10.3389/fphar.2021.592184

\section{Enhanced Expression of Human Epididymis Protein 4 (HE4) Reflecting Pro-Inflammatory Status Is Regulated by CFTR in Cystic Fibrosis Bronchial Epithelial Cells}

\begin{abstract}
Zsolt Bene ${ }^{1,2,3}$, Zsolt Fejes ${ }^{1,3}$, Tibor Gabor Szanto ${ }^{4}$, Ferenc Fenyvesi ${ }^{5}$, Judit Váradi ${ }^{5}$, Luka A. Clarke ${ }^{6}$, Gyorgy Panyi ${ }^{4}$, Milan Macek Jr. ${ }^{7}$, Margarida D. Amaral ${ }^{6}$, István Balogh ${ }^{1,8}$ and Béla Nagy Jr. ${ }^{1,3 *}$

${ }^{1}$ Department of Laboratory Medicine, Faculty of Medicine, University of Debrecen, Debrecen, Hungary, ${ }^{2}$ Department of Pediatrics, Faculty of Medicine, University of Debrecen, Debrecen, Hungary, ${ }^{3}$ Kálmán Laki Doctoral School of Biomedical and Clinical Sciences, Faculty of Medicine, University of Debrecen, Debrecen, Hungary, ${ }^{4}$ Department of Biophysics and Cell Biology, Faculty of Medicine, University of Debrecen, Debrecen, Hungary, ${ }^{5}$ Department of Pharmaceutical Technology, Faculty of Pharmacy, University of Debrecen, Debrecen, Hungary, ${ }^{6}$ Faculty of Sciences, BiolSI-Biosystems and Integrative Sciences Institute, University of Lisboa, Lisboa, Portugal, ${ }^{7}$ Department of Biology and Medical Genetics, Charles University - 2nd Faculty of Medicine and Motol University Hospital, Prague, Czech, ${ }^{8}$ Division of Clinical Genetics, Department of Laboratory Medicine, Faculty of Medicine, University of Debrecen, Debrecen, Hungary
\end{abstract}

Decreased human epididymis protein 4 (HE4) plasma levels were reported in cystic fibrosis (CF) patients under CFTR potentiator ivacaftor therapy, which inversely correlated with lung function improvement. In this study, we investigated whether HE4 expression was affected via modulation of CFTR function in CF bronchial epithelial (CFBE) cells in vitro. HE4 protein levels were measured in the supernatants of CFBE $410^{-}$cells expressing F508delCFTR or wild-type CFTR (wt-CFTR) after administration of lumacaftor/ivacaftor or tezacaftor/ivacaftor, while HE4 expression in CFBE $410^{-}$cells were also analyzed

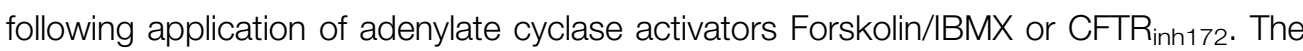
effect of all of these compounds on CFTR function was monitored by the whole-cell patchclamp technique. Induced HE4 expression was studied with interleukin-6 (IL-6) in F508delCFTR CFBE $410^{-}$cells under TNF-a stimulation for $1 \mathrm{~h}$ up to 1 week in duration. In parallel, plasma HE4 was determined in CF subjects homozygous for $p$.Phe508del-CFTR mutation receiving lumacaftor/ivacaftor (Orkambi ${ }^{\circledR}$ ) therapy. NF-kB-mediated signaling was observed via the nuclear translocation of p65 subunit by fluorescence microscopy together with the analysis of IL-6 expression by an immunoassay. In addition, HE4 expression was examined after NF-kB pathway inhibitor BAY 11-7082 treatment with or without CFTR modulators. CFTR modulators partially restored the activity of F508delCFTR and reduced HE4 concentration was found in F508del-CFTR CFBE $410^{-}$cells that

\footnotetext{
Abbreviations: CF, cystic fibrosis; CFBE, cystic fibrosis bronchial epithelial cell; CFFT, Cystic Fibrosis Foundation Therapeutics; CFTR, CF transmembrane conductance regulator; $\mathrm{FEV}_{1}$, forced expiratory volume in 1 second; HE4, human epididymis protein 4; IL-6, interleukin-6; LPS, lipopolysaccharide; NF-KB, nuclear factor-kappa B; PBMCs, peripheral blood mononuclear cells; PEx, pulmonary exacerbation; RT, room temperature; SLPI, Secretory Leukocyte Protease Inhibitor; TNF- $\alpha$, tumor necrosis factor $\alpha$; WFDC, whey acidic protein four-disulfide-core; wt-CFTR, wild-type CFTR.
} 
was close to what we observed in CFBE $410^{-}$cells with wt-CFTR. These data were in agreement with decreased plasma HE4 concentrations in CF patients treated with Orkambi $i^{\circledR}$. Furthermore, CFTR inhibitor induced elevated HE4 levels, while CFTR activator Forskolin/IBMX downregulated HE4 in the cell cultures and these effects were more pronounced in the presence of CFTR modulators. Higher activation level of baseline and TNF- $\alpha$ stimulated NF- $\kappa B$ pathway was detected in F508del-CFTR vs. wt-CFTR CFBE $410^{-}$cells that was substantially reduced by CFTR modulators based on lower p65 nuclear positivity and IL-6 levels. Finally, HE4 expression was upregulated by TNF- $\alpha$ with elevated IL-6, and both protein levels were suppressed by combined administration of NF-kB pathway inhibitor and CFTR modulators in CFBE $410^{-}$cells. In conclusion, CFTR dysfunction contributes to abnormal HE4 expression via NF-kB in CF.

Keywords: cystic fibrosis, inflammation, bronchial epithelial cell, HE4, CFTR modulator

\section{INTRODUCTION}

Cystic fibrosis (CF; MIM:219700) is a monogenic disorder that is caused by pathogenic variants in the cystic fibrosis transmembrane conductance regulator (CFTR) gene (MIM: 602421). CFTR protein is a chloride/bicarbonate channel, which regulates fluid transport across the apical membrane at various epithelial surfaces comprising e.g., the sweat gland, lumen of the bronchial tree and pancreatic exocrine ducts (Rowe et al., 2005). More than 2,000 variants have been identified in the CFTR gene with the major $p$. Phe508del-CFTR allele accounting for approximately $80 \%$ of all CF-causing alleles. CFTR dysfunction leads to impaired ion transport across epithelial surfaces resulting in airway dehydration and thick mucus secretion associated with chronic respiratory bronchial inflammation/obstruction that is further compounded by chronic lung colonization with pathognomonic bacteria, such as $P$. aeruginosa (Rowe et al., 2005). Neutrophil infiltration with high intrapulmonary protease levels (e.g., neutrophil elastase) and excess of proinflammatory cytokines, such as interleukin 6 (IL-6) are associated with "hyperinflammation" in the CF lung leading to the progressive damage of the bronchial tree (Nixon et al., 1998; Cantin et al., 2015).

The human epididymis protein 4 (HE4) is encoded by the WFDC2 gene (MIM:617,548). Its enhanced expression was first detected in the lower, chronically inflamed CF airways according to immunohistochemistry findings (Bingle et al., 2006). Moreover, WFDC2 was reported to be among the upregulated genes in the native CF nasal epithelium (Clarke et al., 2013). Since then, elevated serum HE4 concentration was positively associated with the overall disease severity of $\mathrm{CF}$ and the degree of pulmonary dysfunction in unrelated patient cohorts (Nagy et al., 2016). Additionally, HE4 mRNA levels were significantly higher in CF vs. non-CF airway biopsy specimens (Nagy et al., 2016).

Recently, CF patients treated with CFTR potentiator ivacaftor (IVA) and carrying at least one Class III CFTR CF-causing mutation ( $p . G l y 551 A s p$ ) had lower plasma HE4 concentrations, which also inversely correlated with the improvement of their spirometry parameters (Nagy et al., 2019). Based on these preliminary results, serum or plasma HE4 concentration represents a novel biomarker that may be of value for routine monitoring of CFTR modulating therapy in CF (Bene et al., 2020).

However, the mechanism of abnormal HE4 expression in CF lung epithelial cells has not been investigated as yet. The link between CFTR dysfunction and chronic airway inflammation has been analyzed by former in vitro studies (Vij et al., 2009; Hunter et al., 2010). These data indicated that wild-type CFTR (wtCFTR) has inherent anti-inflammatory properties that suppress baseline and stimulated NF- $\mathrm{KB}$ mediated inflammatory signaling in bronchial epithelial cells, while in CF abnormal CFTR function contributes to generally increased inflammation via disrupted

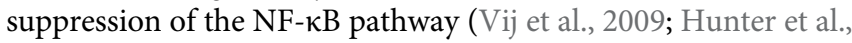
2010). Similarly, aberrant CFTR expression and thus CFTR function reduce the ability of myeloid cells to successfully resolve infection and inflammation (Bonfield et al., 2012).

Introduction of CFTR modulator therapy renders a highly effective therapeutic modality which directly targets the basic CFTR defect and thus substantially improves the overall clinical course of CF (De Boeck and Amaral, 2016). In terms of the effect of CFTR modulators on cellular level of inflammatory processes, lumacaftor/ivacaftor (Orkambi ${ }^{\circledR}$, LUM/IVA) treatment restores CFTR dependent chloride efflux (Favia et al., 2020) and decreases IL-18 and tumor necrosis factor $\alpha$ (TNF- $\alpha$ ) expression in peripheral blood mononuclear cells (PBMCs) when measured in patients homozygous for the p. Phe508del-CFTR allele (JaroszGriffiths et al., 2020). This treatment with LUM/IVA also enhanced airway epithelial repair and thus improved transepithelial resistance, irrespective of the presence of $P$. aeruginosa (Adam et al., 2018). Similarly, tezacaftor/ivacaftor (Symdeko ${ }^{\circledR}$, TEZ/IVA) downregulates serum IL- $1 \beta$ level at 3 months following its patient administration (Jarosz-Griffiths et al., 2020). Finally, in our recent clinical study, treatment with ivacaftor resulted in significantly lower plasma HE4 concentrations in three independent cohorts of CF patients already at 1 month following initiation of therapy (Nagy et al., 2019).

Currently, it is not clear whether HE4 expression is "directly" regulated by CFTR and thus could be influenced by CFTR modulators in vitro using human $\mathrm{CF}$ bronchial epithelial 
(CFBE) cells as a model cell culture system. Therefore, the major aims of this study are i) to determine HE4 level in the supernatants of cystic fibrosis bronchial epithelial (CFBE) $410^{-}$ cells expressing F508del-CFTR or wt-CFTR after in vitro administration of clinically relevant concentrations of CFTR modulators as well as CFTR activators and inhibitor; ii) to analyze HE4 concentrations in plasma samples drawn from CF subjects receiving Orkambi ${ }^{\circledR}$; and iii) to investigate the role of NF- $\kappa B$ pathway in HE4 expression in association with impaired CFTR function and pro-inflammatory signaling in CF. To the best of our knowledge, such comprehensive approaches have not been applied thus far.

\section{MATERIALS AND METHODS}

\section{Reagents}

CFTR correctors lumacaftor (VX-809, LUM) (S1565) and tezacaftor (VX-661, TEZ) (S7059), CFTR potentiator ivacaftor (VX-770, IVA) (S1144), voltage-independent selective CFTR inhibitor CFTR $_{\text {inh172 }}$ (S7139), CFTR activator Forskolin (FSK, S2449), and NF- $\mathrm{BB}$ pathway inhibitor BAY 11-7082 (S2913) were purchased from Selleck Chemicals (Houston, TX, United States). cAMP phosphodiesterase inhibitor IBMX (3-isobuthyl-1methylxanthine, I5879) was ordered from Sigma-Aldrich (St. Louis, MO, United States). Except for recombinant TNF-a (Gibco, Carlsbad, CA, United States), all reagents were dissolved in dimethyl sulfoxide (DMSO, Sigma-Aldrich).

\section{Cell Culture}

CFBE $410^{-}$cells cultures stably expressing F508del-CFTR or wtCFTR were grown in Minimum Essential Medium Eagle (EMEM) with Earle's BSS (EBSS) and 1\% L-glutamine (Lonza, Walkersville, MD, United States), 10\% fetal bovine serum (FBS, Sigma-Aldrich) and $5 \mu \mathrm{g} / \mathrm{ml}$ Puromycin (Sigma-Aldrich) at $37^{\circ} \mathrm{C}, 5 \% \mathrm{CO}_{2}$ (Nagy et al., 2016). These cells were obtained from Dr J. P. Clancy's lab (Cincinnati Children's Hospital Medical Center, OH, United States). CFBE cells were seeded in 6-well plates (250.000 cells per well/ sample). Supernatants for the analysis of HE4 and IL- 6 protein levels were collected after CFBE cells were treated with TNF- $\alpha$ or phosphate buffer solution PBS, (i.e. at baseline) and combined CFTR modulators: corrector lumacaftor $(3 \mu \mathrm{M})$ with potentiator ivacaftor $(10 \mu \mathrm{M})(\mathrm{LUM} / \mathrm{IVA})$ or corrector tezacaftor $(5 \mu \mathrm{M})$ with ivacaftor $(10 \mu \mathrm{M})(\mathrm{TEZ} / \mathrm{IVA})$ or DMSO vehicle alone (thus representing the baseline) were administered for $24 \mathrm{~h}$. For the activation of CFTR function, FSK $(10 \mu \mathrm{M})$ with IBMX $(100 \mu \mathrm{M})$ (FSK/IBMX) were added to both types of CFBE cells, while CFTR inhibition was carried out by $\mathrm{CFTR}_{\text {inh172 }}(20 \mu \mathrm{M})$ in wt-CFTR CFBE cells vs. control samples with DMSO with or without CFTR modulators for $24 \mathrm{~h}$. CFTR modulators were applied under similar experimental conditions as in comparable in vitro studies (Hunter et al., 2010; Wang et al., 2016; Pranke et al., 2017; Kmit et al., 2019). To investigate the role of NF- $\kappa$ B pathway in HE4 expression in vitro, BAY 11-7082 (5 $\mu \mathrm{M})$ or DMSO (baseline) was used for $24 \mathrm{~h}$ to inhibit pro-inflammatory signaling in both unstimulated and TNF- $\alpha$ activated CFBE cells both in the presence or absence of LUM/IVA or TEZ/IVA molecules.

\section{Electrophysiology}

$\mathrm{Cl}^{-}$currents in CFBE $410^{-}$cells were measured in the whole-cell patch-clamp configuration similar to former publications (Boinot et al., 2014; Billet et al., 2017). The external (bath) solution contained $145 \mathrm{mM} \mathrm{NaCl}, 4 \mathrm{mM} \mathrm{CsCl}, 1 \mathrm{mM} \mathrm{CaCl}, 1 \mathrm{mM}$ $\mathrm{MgCl}_{2}, 5 \mathrm{mM}$ D-glucose, and $10 \mathrm{mM}$ HEPES (pH 7.4 titrated with $\mathrm{NaOH}, 315 \mathrm{mOsm}$ ). The intracellular (pipette) solution contained $113 \mathrm{mM}$-aspartic acid, $113 \mathrm{mM} \mathrm{CsOH}, 27 \mathrm{mM} \mathrm{CsCl}$, $1 \mathrm{mM} \mathrm{NaCl}, 1 \mathrm{mM} \mathrm{MgCl}, 1 \mathrm{mM}$ ethylene glycol tetraacetic acid (EGTA), $10 \mathrm{mM}$ HEPES, and $3 \mathrm{mM} \mathrm{Mg-ATP} \mathrm{(pH} 7.2$ titrated with $\mathrm{CsOH}, 285 \mathrm{mOsm})$. Mg-ATP was freshly diluted into the intracellular solution every hour. The intracellular solution was stored on ice before usage. FSK/IBMX and CFTR $_{\text {inh-172 }}$ were freshly diluted into the extracellular solution before the start of the experiments, respectively. Micropipettes were pulled in four stages by using a Flaming Brown automatic pipette puller (Sutter Instruments, San Rafael, CA, United States) from Borosilicate Standard Wall with Filament aluminum-silicate glass (GC150TF10, Harvard Apparatus Co., Holliston, MA, United States) with tip diameters between 0.5 and $1 \mu \mathrm{m}$ and heat polished to a tip resistance ranging typically $3-10 \mathrm{M} \Omega$ in the bath solution. All measurements were carried out by using Axopatch 200B amplifier connected to a personal computer using Axon Digidata 1,550 A data acquisition hardware, respectively (Molecular Devices Inc., Sunnyvale, CA, United States). The holding potential was maintained at $-40 \mathrm{mV}$ throughout the experiments, and two voltage-clamp protocols were used to measure whole-cell CFTR currents. First, a single depolarization from -40 to $0 \mathrm{mV}$ was applied every $5 \mathrm{~s}$ for 4-5 min to monitor the current evolution and to confirm the absence of significant leak current. For determining the current-voltage (I-V) relationship the cells were held at a holding potential of $-40 \mathrm{mV}$ and depolarized to test potentials between -80 and $+80 \mathrm{mV}$ in steps of $20 \mathrm{mV}$ increments every $10 \mathrm{~s}$. Experiments were done at room temperature (RT) ranging between 20 and $24^{\circ} \mathrm{C}$. Data were analyzed using the pClamp10.5 software package (Molecular Devices Inc.). Before analysis, current traces were digitally filtered with a three-point boxcar smoothing filter. Prior to analysis, current traces were corrected for ohmic leak.

\section{Total mRNA Extraction}

Total mRNA from CFBE cell culture samples was isolated by TRI reagent (Molecular Research Center Inc., Cincinnati, $\mathrm{OH}$, United States) according to the manufacturer's recommendations. The purity and the concentration of separated mRNA samples were verified by a NanoDrop 2000 spectrophotometer (Thermo Scientific, Wilmington, DE, United States). Extracted mRNA samples were stored at $-80^{\circ} \mathrm{C}$ before further analysis.

\section{Real-Time Quantitative PCR Analysis}

Complementary DNA (cDNA) synthesis was performed with High Capacity cDNA Reverse Transcription kit (Applied Biosystems, Vilnius, Lithuania) according to the manufacturer's protocol on extracted mRNA samples. The initial amount of RNA was 1,000 ng per reaction. Real-time quantitative PCR (RT-qPCR) was performed on a LightCycler 480 qPCR instrument (Roche Diagnostics, 
Mannheim, Germany) with LightCycler 480 SYBR Green I Master mix (Roche Diagnostics) including WFDC2-specific oligonucleotide primers $(10 \mu \mathrm{M}$, Integrated DNA Technologies, Leuven, Belgium). The reactions were incubated at $95^{\circ} \mathrm{C}$ for $10 \mathrm{~min}$, followed by 40 cycles of $95^{\circ} \mathrm{C}$ for $10 \mathrm{~s}$ and $60^{\circ} \mathrm{C}$ for $1 \mathrm{~min}$. All measurements were run in triplicate. For normalization, we used the reference gene RPLPO (36B4). HE4 expression in TNF-a stimulated CFBE cells was monitored from $1 \mathrm{~h}$ up to 1 week vs. the baseline (using PBS) via measuring mRNA concentrations, while induced cellular activation was followed by IL6-, IL8- and IL1B-specific mRNA levels. Sequences of the primers for respective cDNA amplification are listed in Supplementary Table S1.

\section{Immunofluorescence Staining and Fluorescent Microscopy}

Detection of the NF- $\kappa$ B pathway activation in CFBE $410^{-}$cells with or without CFTR dysfunction was initially visualized via p65 nuclear immunofluorescence staining based on the method drawn from our previous study (Fejes et al., 2018) with some modifications. For this purpose, F508del-CFTR and wt-CFTR CFBE cells were cultured in 12 -well plates on sterile glass microscope slides at a density of $5 \times 10^{4}$ cells/slide for 2 days. Cells were then treated with TNF-a (100 ng/ $\mathrm{mL}$ ) or vehicle (PBS, baseline) for $4 \mathrm{~h}$. When the impact of CFTR modulator treatment on activation level of NF- $\kappa \mathrm{B}$ pathway was studied, CFBE cells were preincubated with lumacaftor $(3 \mu \mathrm{M})$ with ivacaftor $(10 \mu \mathrm{M})$ or tezacaftor $(5 \mu \mathrm{M})$ with ivacaftor $(10 \mu \mathrm{M})$ or DMSO (baseline) for $24 \mathrm{~h}$, and with TNF- $\alpha(100 \mathrm{ng} / \mathrm{ml})$ or PBS (baseline) was added for $4 \mathrm{~h}$. After these pretreatments, cells were fixed with ice-cold methanol-acetone $(50 \mathrm{v} / \mathrm{v} \%)$ for $10 \mathrm{~min}$. Nonspecific antibody binding sites were blocked with fetal bovine serum (FBS, Sigma-Aldrich) for $15 \mathrm{~min}$. For primary labeling of NF- $\kappa$ B p65 subunit, polyclonal rabbit anti-human p65 antibody $(100 \mu \mathrm{g} / \mathrm{ml}$, Sigma-Aldrich) was used for $1 \mathrm{~h}$ followed by secondary staining with Alexa Fluor 488-conjugated goat-anti-rabbit IgG $(5 \mu \mathrm{g} / \mathrm{ml}$, SigmaAldrich) for $1 \mathrm{~h}$. Cell nuclei were labeled with $4^{\prime}, 6$-diamidino-2phenylindole (DAPI, Invitrogen, Carlsbad, CA, United States), and samples were observed by Zeiss Axio Scope. A1 fluorescent microscope (HBO 100 lamp) (Carl Zeiss Microimaging GmbH, Goettingen, Germany). DAPI: excitation at $365 \mathrm{~nm}$, emission BP filter $445 / 50 \mathrm{~nm}$; fluorescein: excitation of BP filter at $470 / 40 \mathrm{~nm}$, emission BP filter 525/50 nm. Images were analyzed with ZEN 2012 v.1.1.0.0. software (Carl Zeiss Microimaging $\mathrm{GmbH}$ ). The ratio of nuclear and perinuclear (cytosol) fluorescence intensity was calculated for NF-kB p65 staining. The specificity of immunostaining was checked by incubating the cells with the secondary antibody only, and where very limited background staining was seen.

\section{CF Patients}

Ten CF patients with the classical and stable form of the disease and being homozygous for the $p$. Phe508del-CFTR pathogenic variant ( 5 females and 5 males, mean age of $16.1 \pm 4.8$ years) were randomly selected from a pre-existing cohort in order to measure plasma HE4 levels in samples obtained at 1 month of Orkambi ${ }^{\circledR}$ administration (Vertex Pharmaceuticals, Boston, MA, United States) (Supplementary Table S2). These subjects formerly participated in the PROSPECT study (ClinicalTrials.gov identifier: NCT0247731), and samples were requested from Cystic Fibrosis Foundation Therapeutics (CFFT) Biorepository (Bethesda, MD, United States). Mean value of baseline forced expiratory volume in $1 \mathrm{~s}\left(\mathrm{FEV}_{1} \%\right.$ predicted) of these subjects was $74.6 \pm 16.6 \%$. Before treatment, mean sweat chloride concentration was $101.2 \pm 9.2 \mathrm{mmol} / \mathrm{L}$, while the mean change of sweat chloride was $-19.6 \mathrm{mmol} / \mathrm{L}$. Aliquots of their blood samples were obtained through venous puncture, and were centrifuged, then stored at $-80^{\circ} \mathrm{C} . \mathrm{K}_{3}$-EDTA anticoagulated plasma aliquots were transferred from CFFT for HE4 analysis by international courier service on dry ice to the Department of Laboratory Medicine, University of Debrecen, Hungary.

\section{Laboratory Analyses}

Chemiluminescent microparticle immunoassay (Architecti1000SR ${ }^{\circledR}$, Abbott Diagnostics, Wiesbaden, Germany) was used to analyze protein levels of HE4 in the supernatants obtained from CFBE cell cultures following different research treatments as indicated above. In addition, HE4 plasma concentrations were measured before treatment and after LUM/IVA treatment with the same immunoassay that we used on our previous cohorts (Nagy et al., 2019). IL-6 levels were measured by electrochemiluminescent immunoassay on a Cobas e 411 instrument (Roche Diagnostics). These measurements were performed in an analyst-blinded mode in all studied cases in order to avoid any potential operator-related bias.

\section{Ethics Statement}

This study was approved by the Regional Ethics Committee of the University of Debrecen (permit number: 4813-2017) in accordance with the World Medical Association Declaration of Helsinki-Ethical Principles for Medical Research Involving Human Subjects.

\section{Statistical Analysis}

The Kolmogorov-Smirnov test was used for the evaluation of the normality of the data. Data are expressed in mean \pm SD or SEM, where applicable. Unpaired $t$-test or Mann-Whitney $U$ test was performed to compare two groups of data, while comparison of multiple groups was performed using the ANOVA with Bonferroni's multiple comparisons test. For comparison of plasma HE4 levels before and under CFTR modulator treatment, paired $t$-test was utilized. The $p<0.05$ probability level was regarded as being statistically significant. Analyses were performed using GraphPad Prism, version 6.01 (GraphPad Software, La Jolla, CA, United States).

\section{RESULTS}

\section{CFTR Modulators Partially Rescue F508del-CFTR $\mathrm{Cl}^{-}$Currents in CFBE 410 cells}

In order to demonstrate that the CFTR modulators applied in this study restore CFTR function in airway epithelial cells in vitro, we treated human CFBE 410-cell cultures expressing F508del-CFTR with 

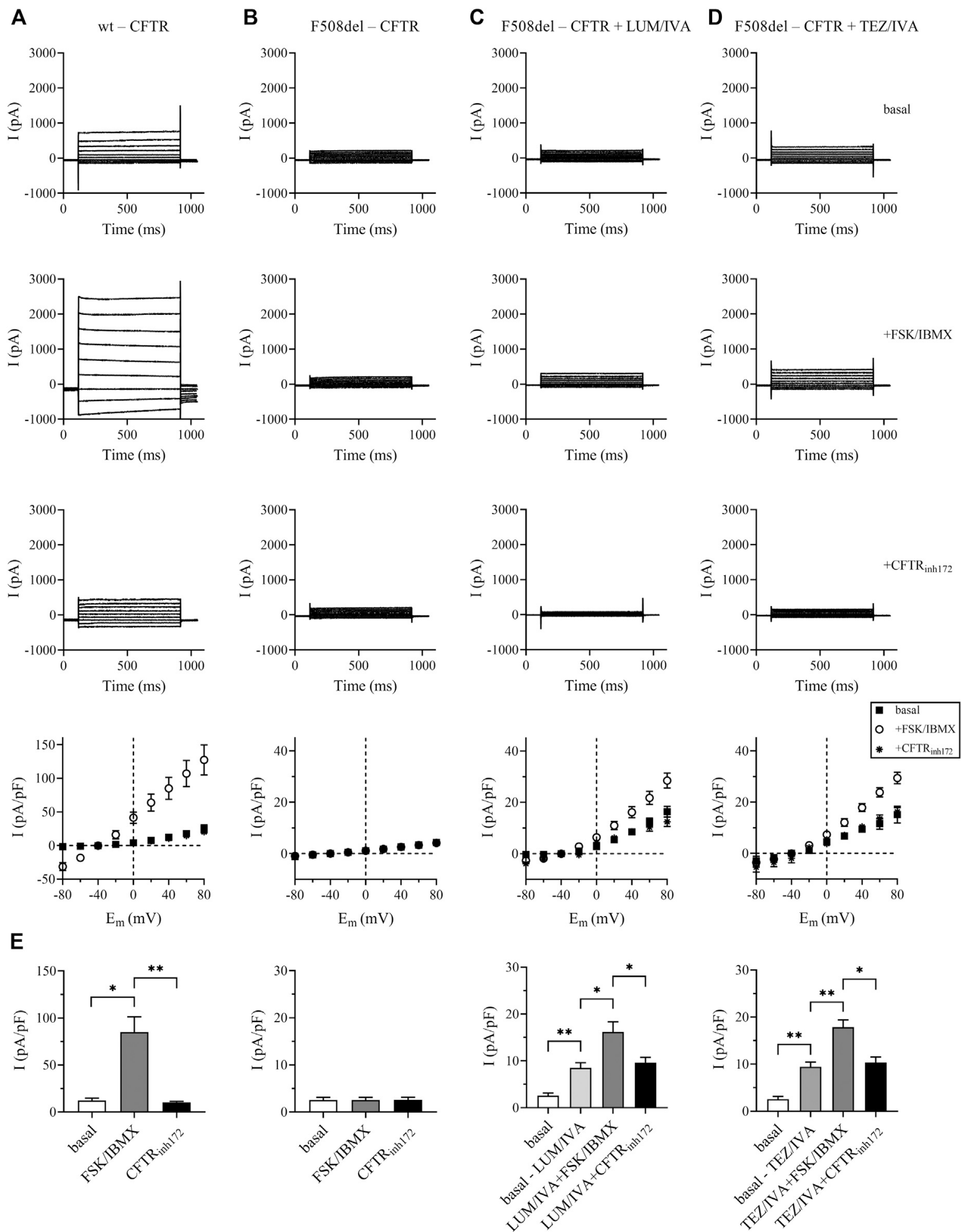

FIGURE 1 | Rescue of functional F508del-CFTR $\mathrm{Cl}^{-}$currents in LUM/IVA and TEZ/IVA-treated CFBE $410^{-}$cells. Representative traces of whole-cell Cl- currents were elicited by stepping from a holding potential of $-40 \mathrm{mV}$ to series test potentials ranging from -80 to $+80 \mathrm{mV}$ with 20 - $\mathrm{mV}$ increments every $10 \mathrm{~s}$ in $\mathrm{CFBE} 41 \mathrm{O}^{-}$cells expressing the wild-type CFTR [wt-CFTR, column (A)], the F508del-CFTR [F508del-CFTR, column (B)], or in cells expressing the deletion mutant CFTR but treated for $24 \mathrm{~h}$ with LUM/IVA [3/10 $\mu \mathrm{M}$, column (C)], or TEZ/IVA [5/10 $\mu \mathrm{M}$, column (D)]. Currents were measured at RT. Top traces: basal current, in the absence of FSK IBMX stimulation; middle traces: after $\sim 2$ min stimulation by FSK/IBMX (10/100 $\mu \mathrm{M})$; bottom traces: in the presence of FSK/BMX and $20 \mu \mathrm{M}$ of the CFTR inhibitor 
FIGURE 1 | CFTR inh172. The bottom panels show the peak current densityvoltage relationships ( $\mathrm{pA} / \mathrm{pF}$, mean $\pm \mathrm{SEM}, \mathrm{n}=3-5)$ in the absence of FSK IBMX (basal, filled squares), upon stimulation by FSK/IBMX (open circles) and in the presence of FSK/IBMX and $20 \mu \mathrm{M} \mathrm{CFTR}_{\text {inh172 }}$ (asterisks). Histograms of the corresponding current densities $(\mathrm{pA} / \mathrm{pF})$ determined at $+40 \mathrm{mV}$. Data are expressed in mean \pm SEM, $n=3-5$ cells/condition (E) Basal: wild-type CFTR expressing cells (left panel) or F508del-CFTR expressing cells (all other panels) in the absence of FSK/IBMX stimulation. Basal-LUM/IVA and basal-TEZ/IVA: F508del-CFTR expressing cells treated with the CFTR modulators only (see above), FSK/IBMX: stimulation of basal or LUM/IVA or TEZ/IVA-treated cells

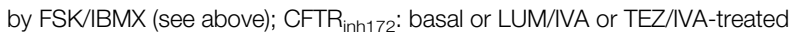

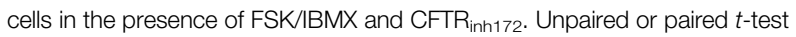
was performed for comparisons. ${ }^{*} p<0.05,{ }^{* *} p<0.01$.

two different combinations of CFTR modulators (LUM/IVA or TEZ/ IVA) for $24 \mathrm{~h}$ and analyzed $\mathrm{Cl}^{-}$current density using patch-clamp. CFBE $410^{-}$cells expressing wild-type CFTR showed whole-cell $\mathrm{Cl}^{-}$ currents that could be robustly activated by FSK/IBMX and inhibited

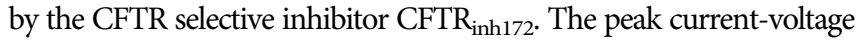
relationship indicated (Figure 1A, bottom panel) a linear currentvoltage relationship, which was most obvious after FSK/IBMX treatment, that reversed around $-40 \mathrm{mV}$, the expected reversal potential of a $\mathrm{Cl}^{-}$current calculated from the ionic composition of the pipette-filling and extracellular solutions. The same experiments in CFBE $410^{-}$cells expressing F508del-CFTR resulted in miniature currents that were insensitive to either FSK/IBMX activation or inhibition by $\mathrm{CFTR}_{\text {inh172 }}$ (Figure 1B). Most importantly, both combinations of CFTR modulators (LUM/IVA, Figure 1C, or TEZ/IVA, Figure 1D) significantly increased the basal and the FSK/IBMX-stimulated $\mathrm{Cl}^{-}$current in comparison to F508delCFTR basal $\mathrm{Cl}^{-}$current (Figures 1B-D). Moreover, the currents activated by FSK/IBMX treatment were sensitive to CFTR $_{\text {inh172 }}$ (Figures 1C,D). The statistical analysis of the current densities at $+40 \mathrm{mV}$ in Figure 1E confirms that CFTR modulators corrected F508del-CFTR channel function (Figure 1E). The current densities recorded in the presence of FSK/IBMX in cells treated with either LUM/IVA or TEZ/IVA were comparable to the wt-CFTR current density in the absence of the activators $(\sim 20 \mathrm{pA} / \mathrm{pF})$ and smaller than wt-CFTR current after stimulation $(\sim 80 \mathrm{pA} / \mathrm{pF})$. Of note, TEZ/IVA restored F508del-CFTR Cl${ }^{-}$current density at a moderately higher level than LUM/IVA $(9.44 \pm 1.01$ vs. $8.48 \pm 1.14 \mathrm{pA} / \mathrm{pF} ; \mathrm{p}=0.560)$. In summary, LUM/IVA and TEZ/IVA CFTR modulators partially restored CFTR function in CFBE $410^{-}$cells cultures expressing F508del-CFTR.

\section{CFTR Function Modulates HE4 Concentrations in CFBE $410^{-}$cells Culture Supernatants in vitro}

CFBE 410-cell cultures expressing F508del-CFTR were treated with CFTR modulators LUM/IVA or TEZ/IVA for $24 \mathrm{~h}$ to quantify protein levels of HE4 in the cell culture supernatants. Subsequently, CFBE $410^{-}$cells with wt-CFTR were utilized as controls for HE4 supernatant concentrations. We consistently found that baseline HE4 concentration was higher in F508del-CFTR CFBE $41 \mathrm{o}^{-}$cells than normal cells $(p<0.01)$ and was significantly reduced by LUM/IVA $(p<$ $0.01)$ and TEZ/IVA $(p<0.001)$ treatment compared to
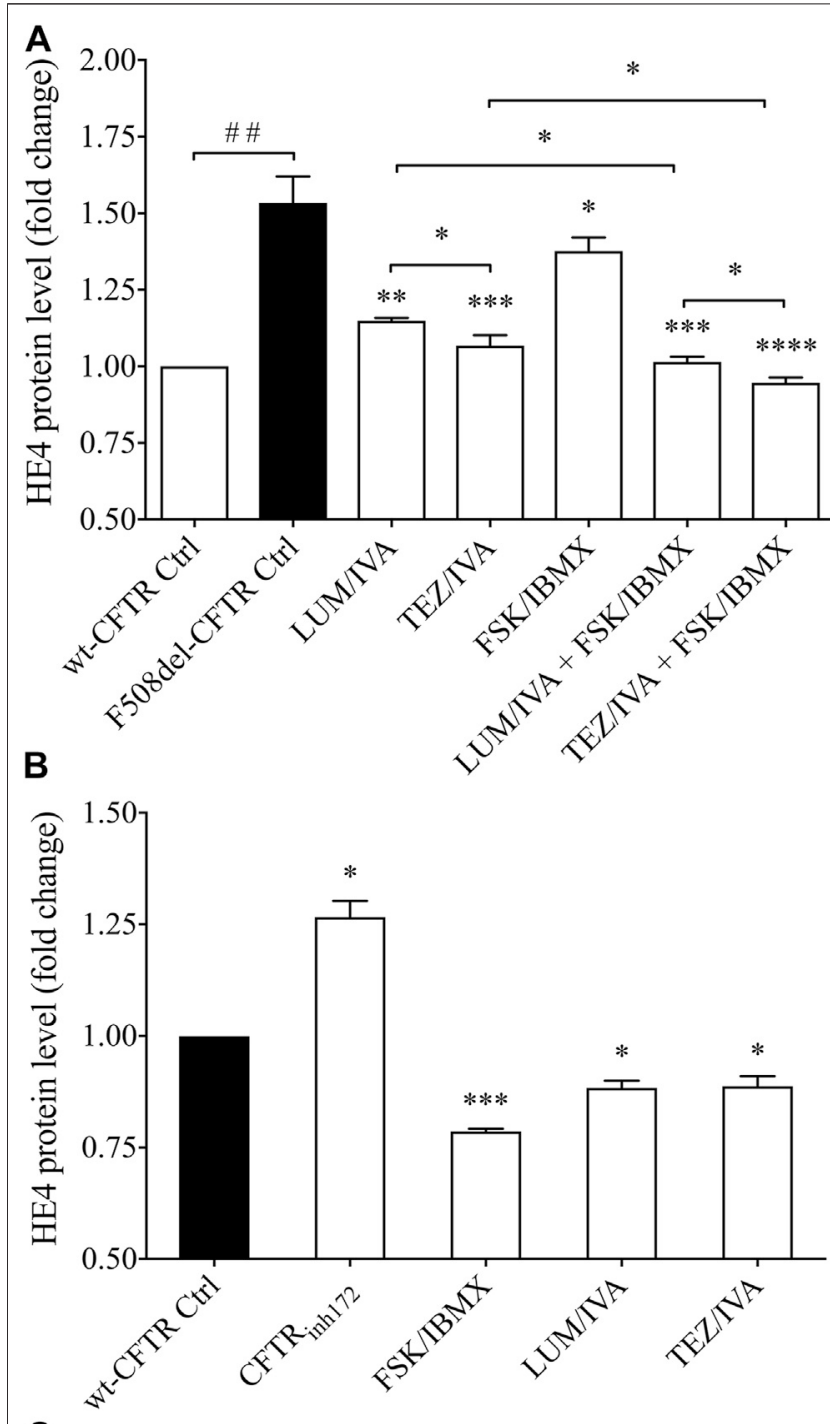

C

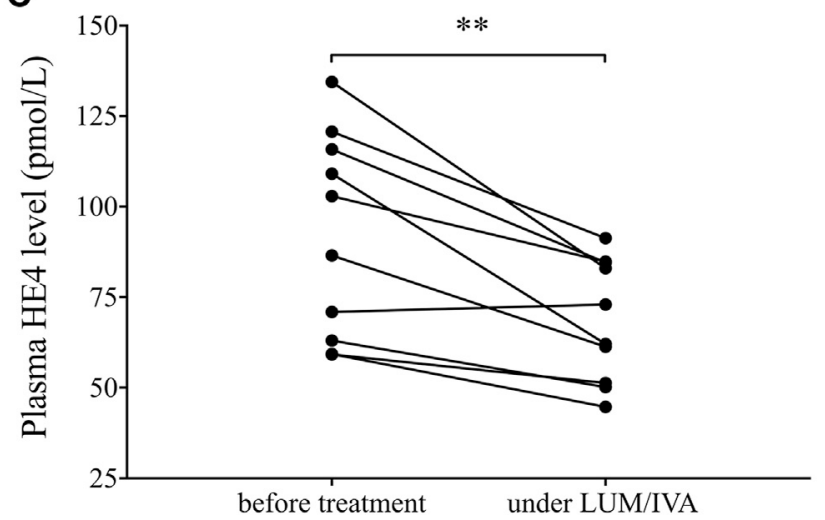

FIGURE 2 | Analysis of HE4 levels in the supernatant of F508del-CFTR or wt-CFTR CFBE $410^{-}$cells with pharmacologically altered CFTR function and in plasma samples of CF subjects under LUM/IVA therapy. Both LUM/IVA $(3 / 10 \mu \mathrm{M})$ and TEZ/VA $(5 / 10 \mu \mathrm{M})$ resulted in decreased HE4 in F508delCFTR CFBE $410^{-}$cells after $24 \mathrm{~h}$ approaching the level of normal cells. CFTR activator FSK/IBMX (10/100 $\mu \mathrm{M})$ alone moderately reduced HE4, while combined treatment of FSK/BMX with CFTR modulators showed an even

(Continued) 
FIGURE 2 | larger change in HE4 (A) CFTR inh172 $_{2}(20 \mu \mathrm{M})$ caused elevated HE4 levels after $24 \mathrm{~h}$ while, in turn, improved CFTR function with FSK/IBMX or CFTR modulator reduced HE4 even in wt-CFTR CFBE $410^{-}$cells (B) There were lower plasma HE4 values compared to baseline measured in CF subjects $(n=10)$ after 1 month of Orkambi $^{\circledR}$ in agreement with in vitro data with LUM/IVA (C) Data are expressed in mean \pm SEM ( $n=5-6$ samples/ condition). Unpaired or paired $t$-test or Mann-Whitney $U$ test was performed for comparisons. ${ }^{\# \#} p<0.01$ vs. wt-CFTR cells; ${ }^{\star} p<0.05,{ }^{\star \star} p<0.01,{ }^{\star \star \star} p<$ 0.001 , and ${ }^{\star \star \star *} p<0.0001$ vs. F508del-CFTR control (ctrl) cells with DMSO, or baseline plasma samples, respectively.

control samples where the vehicle (i.e., DMSO) was applied. The concentration of HE4 in the supernatant was close to that observed in wt-CFTR CFBE $410^{-}$cells (Figure 2A). Interestingly, TEZ/IVA caused a larger decrease of HE4 concentrations compared to LUM/IVA $(p<0.05)$. In parallel, CFTR activator FSK/IBMX was used alone and in combination with aforementioned CFTR modulators in cells with F508del-CFTR. The HE4 protein level was decreased by FSK/IBMX $(p<0.05)$ likely due to the activation of residual CFTR function, while further reduction in HE4 was observed after the combined treatment $(p<0.05)$ vs. using individual CFTR modulator molecules (Figure 2A).

To provide further evidence of the inverse association between HE4 expression measured by its concentration in cell culture supernatants and CFTR function, we also applied pharmacological inhibition of CFTR by CFTR $_{\text {inh172 }}$ in wt-CFTR CFBE 410 ${ }^{-}$cells, which caused elevated HE4 levels $(p<0.05)$. In contrast, there was a significant decrease in HE4 concentrations $(p<0.001)$ after FSK/IBMX treatment when compared to the controls. Interestingly, LUM/IVA and TEZ/ IVA could downregulate HE4 expression to a certain extent even in CFBE $410^{-}$cells with normal CFTR expression $(p<$ 0.05) (Figure 2B). These data suggest that CFTR function affects basal levels of HE4 expression and impaired function of CFTR could explain elevated HE4 concentration in CF airway epithelial cells in vitro.

\section{Treatment With LUM/IVA Lowers Plasma HE4 Levels in CF Subjects Homozygous for p.Phe508del-CFTR Mutation}

To substantiate in vivo our in vitro results above, we determined plasma HE4 levels in 10 randomly selected CF individuals homozygous for $p$. Phe508del-CFTR mutation who were under Orkambi ${ }^{\circledast}$ (LUM/IVA) medication. In the presence of decreasing sweat chloride concentrations (Supplementary Table S2), there were significantly $(p<0.01)$ reduced HE4 plasma concentrations-regardless of its baseline value-already at 1 month of treatment, this being the earliest follow-up time point of these patients (Figure 2C). These clinical data underscore the impact of CFTR modulation therapy on decreasing HE4 plasma concentrations in $\mathrm{CF}$ and are in agreement with previous findings in IVA monotherapy in cases with at least one $p$. Gly551Asp-CFTR pathogenic variant (Nagy et al., 2019).

\section{TNF-a Induces Increased HE4 mRNA Expression in CFBE $410^{-}$cells Cultures in vitro}

Subsequently, we studied whether WFDC2/HE4 expression could be further enhanced by an artificial inflammatory stimulus in vitro. For this purpose, F508del-CFTR CFBE $410^{-}$cells were stimulated with recombinant TNF- $\alpha$ applied in the range spanning from $1 \mathrm{~h}$ up to 1 week. As a result, HE4 mRNA level quantified by RT-qPCR raised already after $1 \mathrm{~h}$ of treatment vs. untreated (baseline) sample $(p<0.05)$ and was further elevated within the period of $4 \mathrm{~h}(p<0.001)$. Surprisingly, HE4 mRNA levels returned to baseline within $24 \mathrm{~h}$. When TNF-a was administered for longer periods (from $48 \mathrm{~h}$ up to 1 week), there was a much higher expression of HE4 mRNA $(p<$ 0.0001 ) in TNF-a stimulated CFBE $410^{-}$cells (Figure 3A).

In the same set of samples, mRNA levels of proinflammatory cytokines IL-6, IL- 8 and IL- $1 \beta$ (Figures 3B-D) were also analyzed to ascertain if these mediators were also provoked by TNF-a together with increased expression of HE4. In this regard, IL-6 and IL-8 expression showed similar time-dependent alteration patterns as observed in HE4 mRNA, while elevated IL1B mRNA was sustained from $1 \mathrm{~h}$ without a substantial change throughout this time period. Furthermore, the protein concentrations of HE4 and IL- 6 were measured in the supernatants of F508delCFTR CFBE $41 \mathrm{o}^{-}$cells at some selected time points (between 4 and $168 \mathrm{~h}$ ), whereby HE4 level was significantly elevated after $4 \mathrm{~h}$ and gradually increased up to 1 week of treatment, while IL- 6 concentration was significantly augmented at all pre-selected time points (Supplementary Figures S1A,B). These results imply that expression of HE4 mRNA thus resulting concentrations of HE4 protein are upregulated following TNF- $\alpha$ administration accompanied by different pro-inflammatory cytokines in CFBE cells.

\section{The Pro-inflammatory NF-кB Pathway is Influenced by the Combination of CFTR Modulators in F508del-CFTR CFBE 410 cells in vitro}

To establish the relationship between upregulated NF- $\kappa \mathrm{B}$ pathway due to CFTR dysfunction and the abnormal HE4 expression in CFBE cells in vitro, we applied two experimental approaches. First, the activity of NF- $\kappa B$ pathway was assessed via the p65 nuclear translocation experiments. This protein, also known as RelA (MIM: $164,014)$, is one of the 5 components that constitute the $\mathrm{NF}-\mathrm{\kappa B}$ transcription factor family and is used as a marker of NF- $\kappa B$ pathway activation. We applied fluorescence microscopy-based approaches in wt-CFTR and F508delCFTR CFBE $41 \mathrm{o}^{-}$cells that had been treated with TNF- $\alpha$ vs. untreated controls. Second, the effect of CFTR modulators was studied in vitro on basal and induced NF- $\kappa B$ signaling in these CFBE cells after the application of LUM/IVA or TEZ/ IVA treatment via p65 nuclear positivity and IL- 6 in the cell 

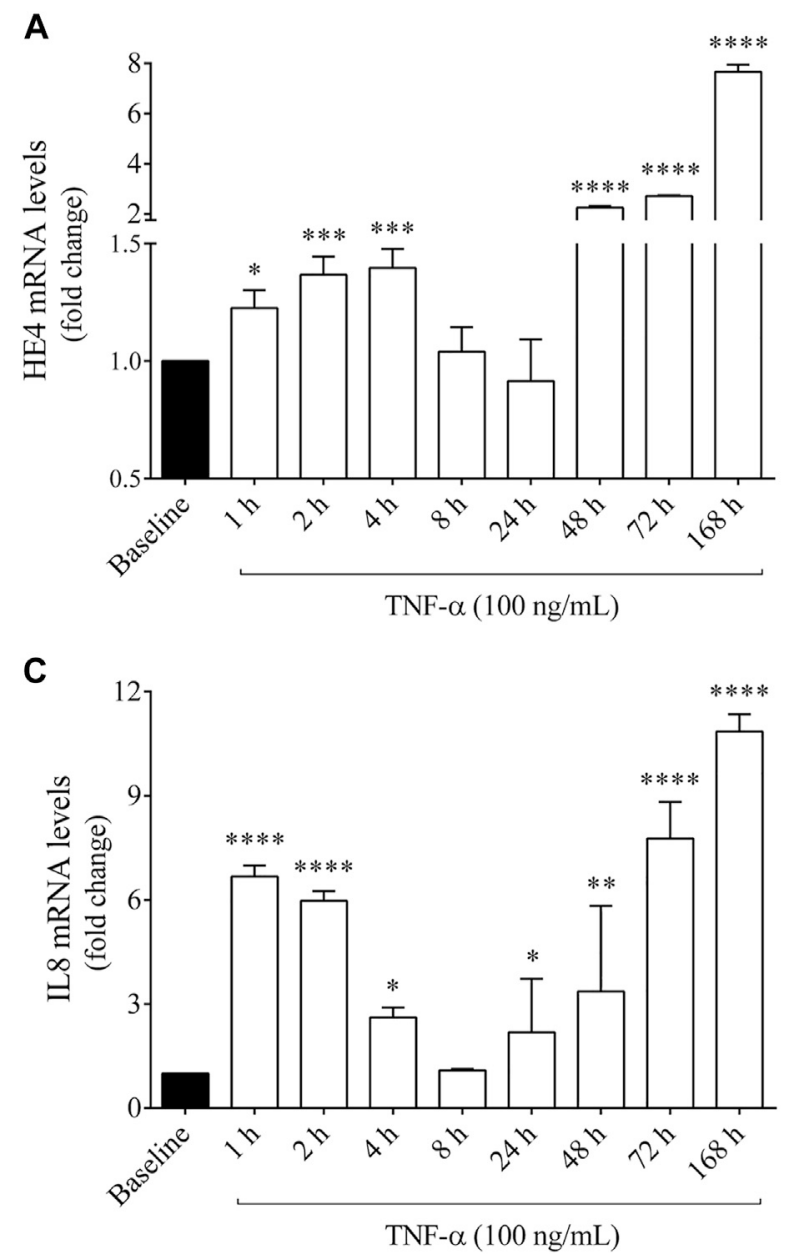
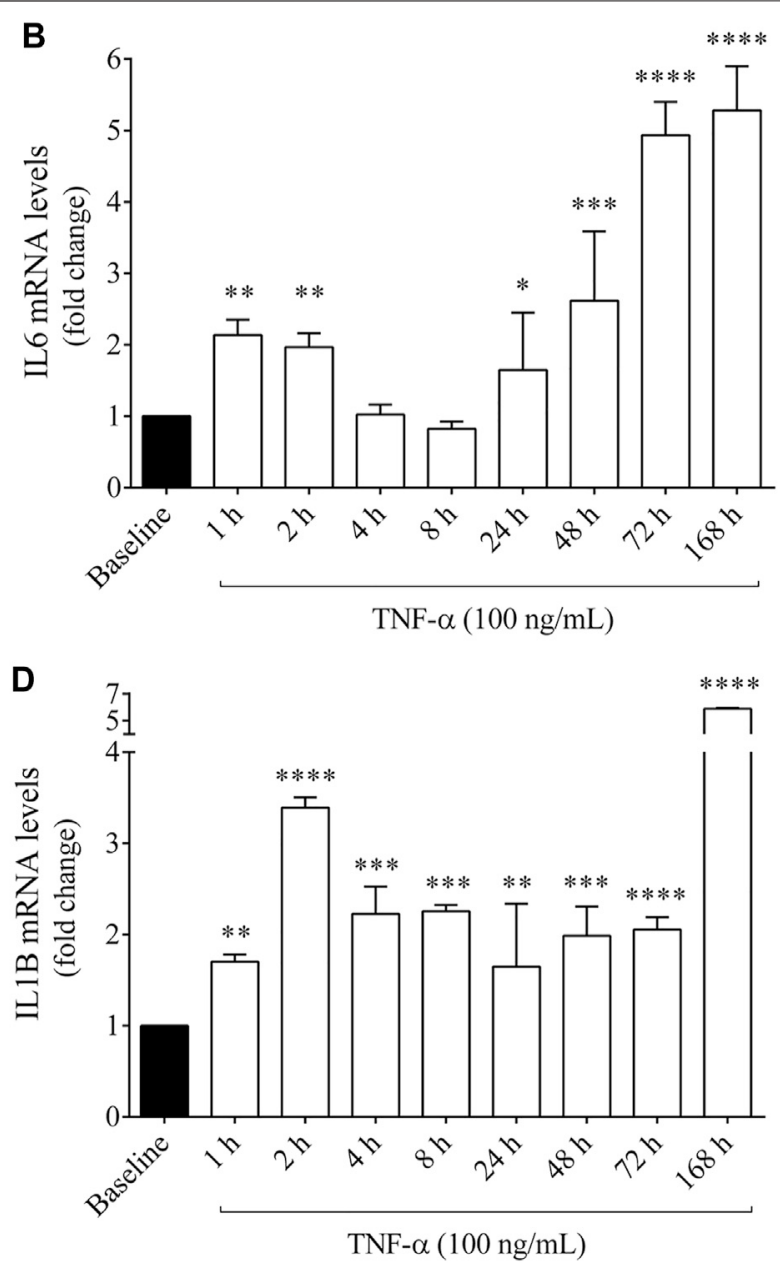

FIGURE 3 | Quantification of HE4, IL6, IL8 and IL1B mRNA levels in F508del-CFTR CFBE 410- cells after TNF-a stimulation for 1 h up to 1 week in vitro. HE4 mRNA level was higher than baseline at $1-4 \mathrm{~h}$ that returned to normal range by $24 \mathrm{~h}$ and was further induced by TNF- $\alpha$ (100 $\mathrm{ng} / \mathrm{ml})$ after $48 \mathrm{~h}$ up to $168 \mathrm{~h}$ (A) IL6 $\mathrm{mRNA}$ level was already induced by 1-2 h and was further augmented after $24 \mathrm{~h}$ (B) In parallel, IL-8 expression showed a similar time-course alteration to HE4 and IL6 (C), while IL1B mRNA level had a sustained elevation without a substantial alteration during this time period (D) Mean \pm SEM ( $n=4-5$ samples/condition). ${ }^{*} p<0.05,{ }^{* *} p<0.01$, $p<0.001$, and ${ }^{\star \star \star \star} p<0.0001$ vs. baseline, based on ANOVA with Bonferroni's multiple comparison test.

line supernatants. There was a significantly higher baseline level of p65 positivity in the nuclei of F508del-CFTR CFBE $410^{-}$cells vs. normal cells $(p<0.01)$. When these cell cultures were exposed to TNF- $\alpha$, significantly higher p65 positivity was seen in both cell types ( $p<0.05, p<0.0001$, respectively), and the difference in p65 translocation between normal and deficient CFBE cells was more pronounced $(p<0.0001)$ (Figures 4A,B). These results indicate that there is a higher baseline and induced level of inflammatory status in CF vs. normal CFBE cells. Secondly, CFTR modulators substantially decreased p65 positivity $(p<0.05)$ not only in unstimulated F508del-CFTR CFBE $41 \mathrm{o}^{-}$cells, but a significant reduction was also observed $(p<0.001)$ after TNF- $\alpha$ treatment compared to baseline (DMSO) sample (Figures 5A,B). In parallel, we determined IL-6 protein levels in the supernatants of studied CFBE cell lines which confirmed the anti-inflammatory effect of applied CFTR modulators via downregulation of basal and TNF- $\alpha$ stimulated IL-6 expression (Figure 5C). Hence, our data provide evidence that the generally increased levels of NF$\kappa \mathrm{B}$ pathway activation due to CFTR dysfunction could be efficaciously downregulated by the application of LUM/IVA or TEZ/IVA in CFBE cell cultures in vitro.

\section{HE4 Concentrations in CFBE $410^{-}$cells Cultures Are Not Only Upregulated by the NF-kB Pathway and Pro-Inflammatory Signaling but Are Also Directly Influenced by CFTR Activity}

Finally, we wanted to examine if TNF- $\alpha$ induced HE4 levels could also be decreased by applying CFTR modulators in F508delCFTR CFBE $410^{-}$cells. Administration of either LUM/IVA or TEZ/IVA resulted in a significant reduction in HE4 concentration $(p<0.001)$ when measured in the supernatants of TNF- $\alpha$ activated CFBE $410^{-}$cells vs. controls with TNF- $\alpha$ and 


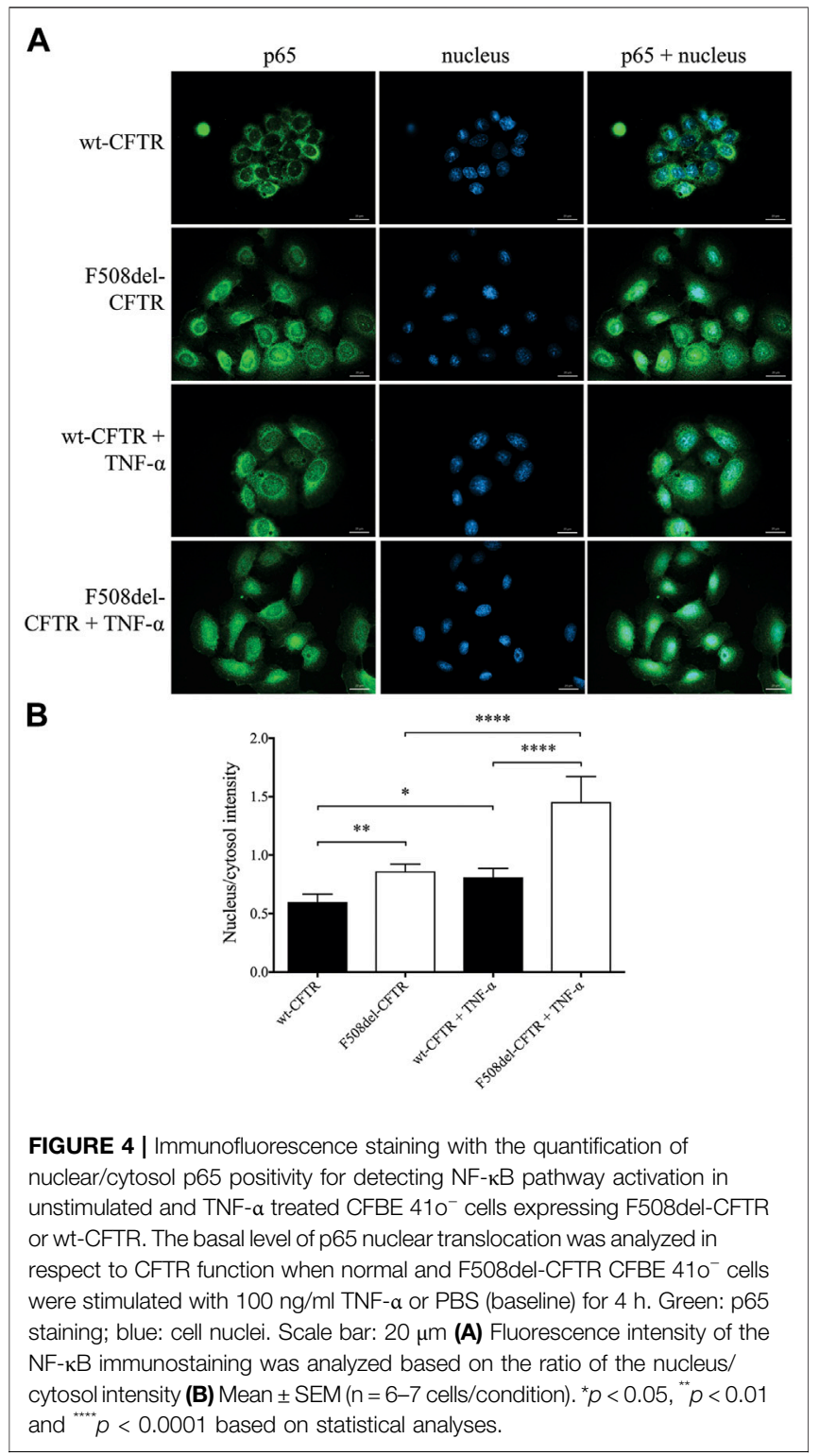

DMSO (Figure 6A). Furthermore, FSK/IBMX alone caused a moderate but still significantly reduced HE4 level in the presence of TNF- $\alpha(p<0.05)$, whereas combined administration of LUM/ IVA or TEZ/IVA with FSK/IBMX lowered HE4 concentrations by a significantly higher degree $(p<0.01 ; p<0.001$, respectively). Similar to the unstimulated samples (Figure 2A), TEZ/IVA caused a larger change in HE4 compared to LUM/IVA with or without CFTR activator $(p<0.05)$. (Figure 6A). When TNF- $\alpha$ activated wt-CFTR epithelial cells were treated with CFTR activator FSK/IBMX or CFTR modulators, there were similarly reduced HE4 levels $(p<0.01)$ compared to TNF- $\alpha$ activated samples with vehicle (Figure 6B). Thus, observed data provide evidence that corrected and/or potentiated CFTR function has a "protective role" against TNF- $\alpha$-induced upregulation of HE4 expression in CFBE cells in vitro.

As we consistently detected enhanced levels of HE4 in the presence of abnormal CFTR function in CFBE $410^{-}$cells bearing
F508del-CFTR in contrast to wt-CFTR cells under non-activated and TNF- $\alpha$ activated conditions, we raised the question whether increased HE4 expression in $\mathrm{CF}$ is under the regulation of NF- $\kappa \mathrm{B}$ mediated pathway directly via impaired CFTR function and related pro-inflammatory stimuli. For this purpose, pretreatment with specific NF- $\kappa \mathrm{B}$ pathway inhibitor BAY 117082 was used in F508del-CFTR CFBE $410^{-}$cells in the absence or presence of CFTR modulators and TNF- $\alpha$ treatment. In these cell culture samples, supernatant HE4 and IL-6 protein levels were determined. We found that BAY 11-7082 mediated inhibition alone significantly lowered baseline HE4 $(p<0.05)$ and IL-6 levels $(p<0.01)$ after $24 \mathrm{~h}$, while BAY 11-7082 with LUM/IVA or TEZ/IVA resulted in a much stronger reduction in the levels of both proteins ( $p<0.001 ; p<0.0001$, respectively) (Figures 7A,B). TNF- $\alpha$-induced HE4 expression was also hindered to a large degree via BAY 11-7082-related inhibition of the NF- $\kappa$ B pathway $(p<0.05)$. Moreover, we noted further decrease in HE4 and IL-6 supernatant concentrations when NF$\kappa \mathrm{B}$ pathway inhibitor and CFTR modulators were administered together vs. control samples with TNF- $\alpha$ and DMSO $(p<0.001)$ (Figures 7A,B). In turn, CFBE $410^{-}$cells with wt-CFTR were treated with CFTR $_{\text {inh172 }}$ with or without BAY 11-7082. In contrast to increased HE4 supernatant concentrations, $\mathrm{CFTR}_{\text {inh172}}$-based inhibition of CFTR was not associated with elevated HE4 concentrations in the supernatant in the presence of the NF- $\kappa \mathrm{B}$ pathway inhibitor $(p<0.05)$ (Figure 7C). Detected changes in IL-6 supernatant concentrations had similar patterns which underscores the close association between CFTR dysfunction and the generally increased pro-inflammatory status in CF (Figure 7D). In summary, HE4 concentrations measured in the supernatants are not only modulated via the NF- $\kappa \mathrm{B}$ pathway and pro-inflammatory signaling, but also directly influenced by CFTR in CFBE cell cultures in vitro.

\section{DISCUSSION}

In this study, we have provided evidence that enhanced in vitro expression of HE4 is regulated by CFTR in CFBE cells. Our results add to the increasing line of evidence that CFTR is not only one of the critical regulators of epithelial fluid balance across various apical epithelial membranes, but may also modulate inflammatory signaling (Eidelman et al., 2001). We have recently reported high concentration of HE4 in serum samples accompanied with its elevated expression in airway epithelial biopsy specimens of $\mathrm{CF}$ individuals (Nagy et al., 2016). In addition, HE4 level was around two-fold higher in the supernatant of F508del-CFTR vs. wt-CFTR CFBE cells (Nagy et al., 2016). HE4 or WFDC2 protein belongs to the whey acidic protein four-disulfide-core (WFDC) protein family, and its members, such as Secretory Leukocyte Protease Inhibitor (SLPI) and Elafin (PI3) possess anti-protease, anti-bacterial and antiinflammatory properties (Small et al., 2017). Due to their abundance in the lung, they have been proposed to be involved in lung homeostasis and protection of the lung from proteolytic "attacks" (Bingle et al., 2006; Small et al., 2017). On the other hand, the mechanism of increased production of HE4 in CF airway epithelial cells is yet unknown. 
A

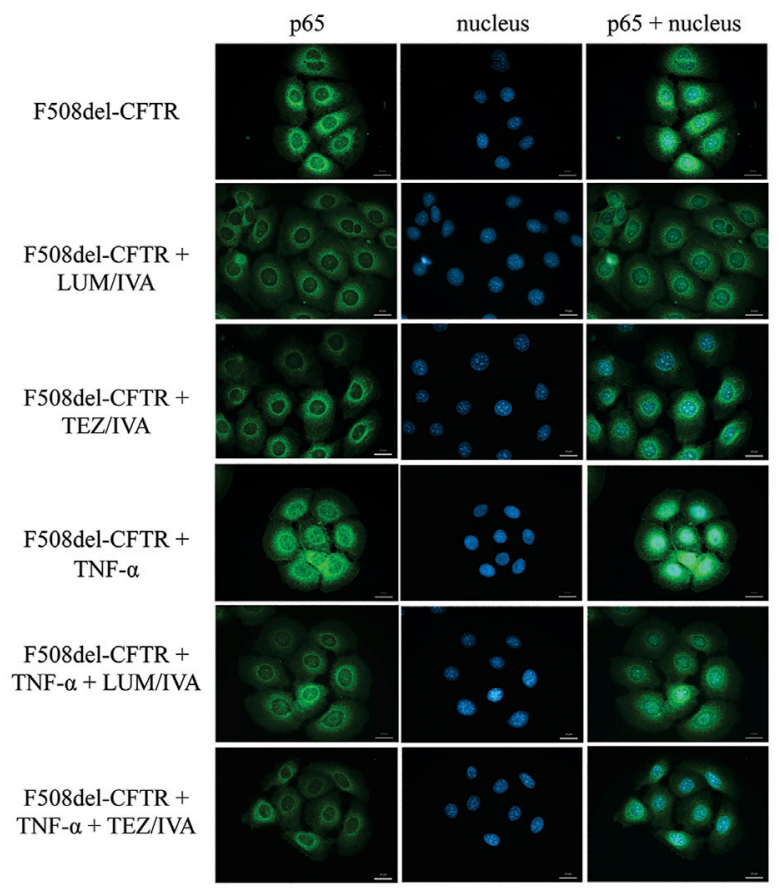

B

C
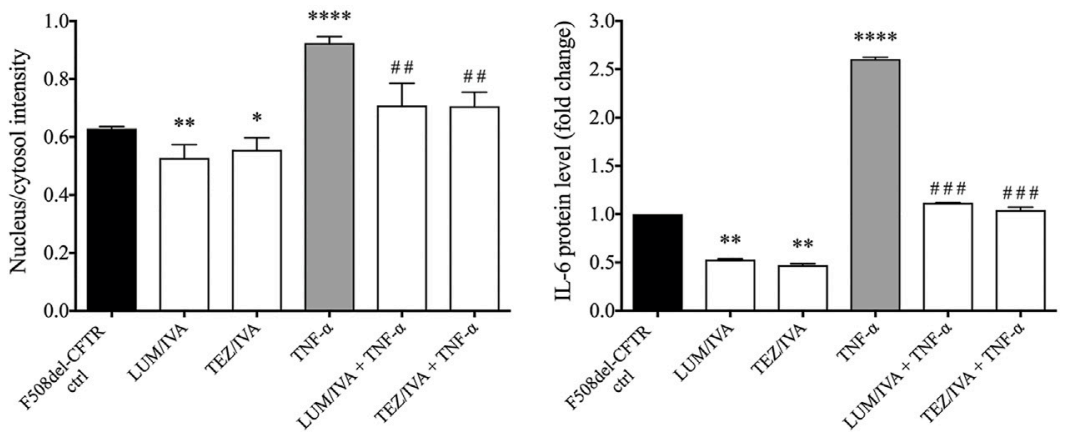

FIGURE 5 | Immunofluorescence staining and quantification of p65 nuclear translocation staining with the analysis of IL-6 protein levels in the presence of CFTR modulators in unstimulated or TNF- $\alpha$ treated CFBE $410^{-}$cells cultures expressing F508del-CFTR. Alteration in p65 nuclear translocation was first analyzed when cells were pretreated with LUM/IVA or TEZ/IVA or DMSO (baseline) for $24 \mathrm{~h}$. CFBE cells were then activated with $100 \mathrm{ng} / \mathrm{ml}$ TNF- $\alpha$ or PBS (baseline) for $4 \mathrm{~h}$. Green: p65 staining; blue: cell nuclei. Scale bar: $20 \mu \mathrm{m}$ (A) Fluorescence intensity of the NF-кB immunostaining was analyzed based on the ratio of the nucleus/cytosol intensity (B) Downregulated IL-6 protein levels were measured in the supernatants of untreated and TNF- $\alpha$ stimulated CFBE 410 -cells to approve the inhibition of NF- $k B$ pathway activation by CFTR modulators by $24 \mathrm{~h}$ (C) Mean \pm SEM, $\mathrm{n}=4-5$ cells/condition. ${ }^{*} p<0.05,{ }^{* *} p<0.01$, and ${ }^{* \star *} p<0.0001$ vs. control cells with DMSO; ${ }^{* \#} p<0.01$ and $\#$ \#\# 0.001 vs. CFBE cells with TNF- $\alpha$ and DMSO.

To date, it is still unclear whether CFTR modulators influence airway inflammatory response via moderating abnormal innate immunity or by epithelial cell activation (Hisert et al., 2017). Latest in vitro investigations described downregulated IL- $1 \beta$ and IL-18 levels derived from lipopolysaccharide (LPS)-stimulated monocytes after LUM/IVA or TEZ/IVA treatment (JaroszGriffith et al., 2020), reduced secretion of pro-inflammatory cytokines with restored macrophage function in response to $P$. aeruginosa after single IVA or combined LUM/IVA therapy (Barnaby et al., 2018), and depressed CXCL8 expression and p38 MAPK phosphorylation in CFBE cells in response to Orkambi ${ }^{\circledast}$ treatment (Ruffin et al., 2018). Accordingly, CFTR corrector and potentiator drugs may have anti-inflammatory capacities through rescued CFTR function and reduced sweat chloride concentration (Ramsey et al., 2011; Hisert et al., 2017). Of note, this is only indirect evidence from clinical trials and postmarketing studies that has not been duly validated in vitro. Moreover, these drugs were not always clinically effective in terms of sustained decrease of chronic bronchial inflammation based on studied biomarkers in the sputum (Rowe et al., 2014), and their anti-inflammatory properties could even gradually vanish after $36 \mathrm{~h}$ of treatment (Jarosz-Griffith et al., 2020). In this regard, previous studies reported that the general level of inflammation is increased via constitutive activation of the NF- $\kappa B$ 

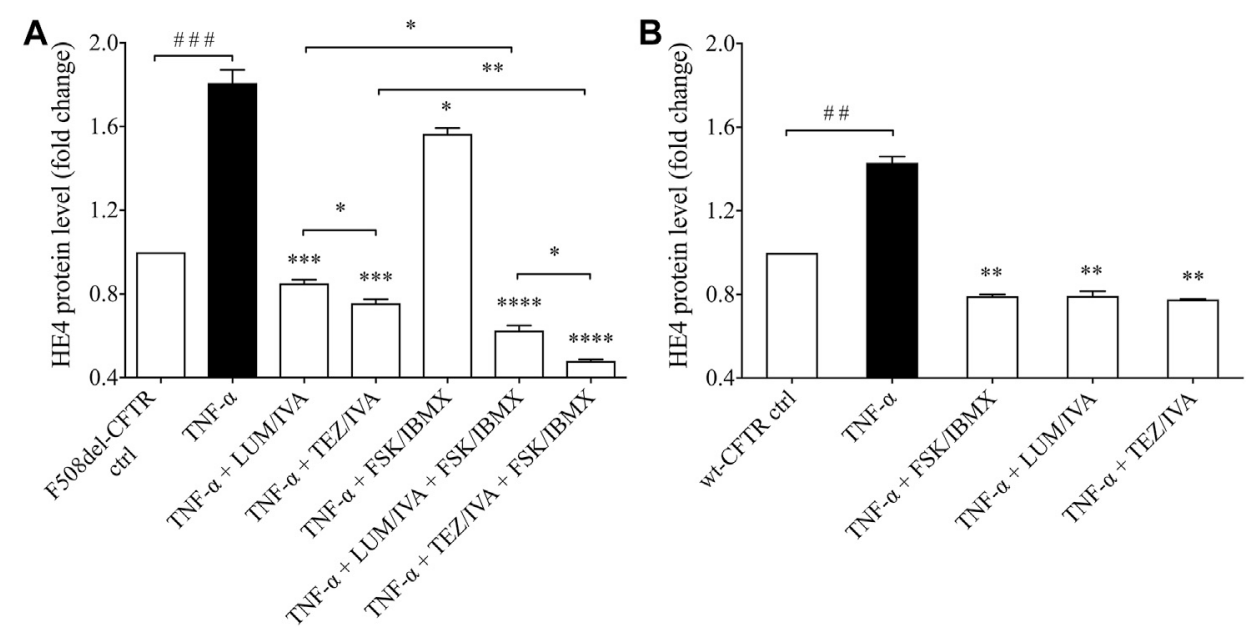

FIGURE 6 | Determination of HE4 level in TNF- $\alpha$ activated F508del-CFTR or wt-CFTR CFBE $410^{-}$cells with in vitro modulated CFTR function. First, TNF- $\alpha$ activated F508del-CFTR CFBE $410^{-}$cells were analyzed under the treatment with LUM/IVA, TEZ/IVA, FSK/IBMX or their combination for 24 h. TNF- $a$ could not elevate HE4 expression in the presence of improved CFTR function (A) In parallel, CFTR function was affected by FSK/BMX or CFTR modulators in TNF- $\alpha$-stimulated wt-CFTR CFBE $410^{-}$cells for $24 \mathrm{~h}$. Similar to the results in F508del-CFTR CFBE $410^{-}$cells, HE4 expression remained lowered compared to samples with TNF- $\alpha$ but with a smaller extent (B) Mean \pm SEM, $n=5-6$ samples/condition. ${ }^{*} p<0.05,{ }^{* *} p<0.01,{ }^{* \star *} p<0.001$ and ${ }^{* \star *} p<0.0001$ vs. control samples with TNF- $\alpha$ and DMSO; ${ }^{\# \#} p<0.01$ and $\#$ \#\#\# 0.001 vs. control samples with PBS.

pathway in CF due to CFTR dysfunction that drives higher production of pro-inflammatory cytokines (e.g. IL-8) even in the absence of pathognomonic infectious agents (Vij et al., 2009; Hunter et al., 2010; Wang et al., 2016). Recent clinical studies provide evidence that administration of CFTR modulators normalizes sweat chloride concentration together with a substantial correction of CFTR function in majority of treated subjects (Ramsey et al., 2011; Wainwright et al., 2015; TaylorCousar et al., 2017).

Therefore, in this study, we aimed to comprehensively analyze how in our opinion promising biomarker HE4 could be influenced by the administration of CFTR modulators, since the pathogenesis of upregulated HE4 expression in CF is not fully understood. Furthermore, we aimed to provide evidence how treatment with CFTR correctors and potentiators could be monitored using HE4 as a plasma biomarker in routine clinical practice and what the particular diagnostic value of HE4 is in monitoring degree of decreased inflammation in CF patients due to restored function of CFTR. Consequently, we investigated for the first time whether abnormal HE4 expression is directly linked to CFTR dysfunction and is associated with NF- $\kappa B$ pathway in $\mathrm{CF}$. We took aforementioned clinical and laboratory evidence into account when designing our methodological approaches.

For this purpose, we analyzed the change in basal and TNFa-induced HE4 levels when CFTR function and NF- $\kappa B$ signaling were pharmacologically modulated in $\mathrm{CFBE} 41 \mathrm{o}^{-}$cells cultures expressing F508del-CFTR in comparison to cells with wt-CFTR. First, there was about 1.5-fold higher basal HE4 concentration in F508del-CFTR vs. wt-CFTR CFBE $410^{-}$cells. Both LUM/IVA and TEZ/IVA significantly lowered HE4 in these cells with F508del-CFTR compared to control samples with vehicle. These compounds were formerly tested for the correction of CFTR in CF epithelial cell cultures via the measurement of CFTR activity in vitro (Pranke et al., 2017; Kmit et al., 2019). Here, the rescue of CFTR function by CFTR modulators was observed with whole-cell patch-clamp technique similar to a former publication (Boinot et al., 2014). Based on our data, we considered F508delCFTR function partially rescued, however, Boinot et al. suggested that if the current density was greater than $4 \mathrm{pA} / \mathrm{pF}$ after treatment with CFTR modulator, the CFTR activity was corrected (Boinot et al., 2014). As a consequence, HE4 was significantly reduced by both combinations of CFTR modulators. Importantly, TEZ/IVA caused a stronger alteration in both $\mathrm{Cl}^{-}$current and $\mathrm{HE} 4$ concentrations compared to LUM/IVA.

Treatment with IVA previously lowered plasma HE4 levels in CF patients bearing a specific Class 3 CFTR pathogenic variant (Nagy et al., 2019), but no data were available on the impact of combined therapy in vivo with various CFTR correctors and modulators on plasma HE4 as yet. Hence, we determined plasma HE4 levels in 10 CF individuals homozygous for $p$. Phe508del-CFTR mutation who were taking LUM/IVA regimen. In accordance with in vitro data, we provided evidence that this combination of CFTR modulators (Orkambi ${ }^{\circledR}$ ) led to significantly decreased HE4 plasma concentrations, regardless of individual baseline HE4 concentrations and already after 1 month since the initiation of treatment. Subsequently, we used pharmacological inhibition of CFTR by CFTR $_{\text {inh172 }}$ in CFBE $410^{-}$cells with wt-CFTR that caused elevated HE4 levels in vitro. In contrast, there was a significant decrease in HE4 concentrations after CFTR activator FSK/IBMX treatment in wt-CFTR cells compared to controls. These data are in accordance with previous reports on elevated NF- $\kappa B$ activity with higher IL-8 level following CFTR $_{\text {inh172 }}$ administration and decreased NF- $\kappa$ B activity after FSK/IBMX treatment in another cell culture (Hunter et al., 2010). In addition, FSK/IBMX treatment resulted in a moderate but statistically significant reduction of HE4 


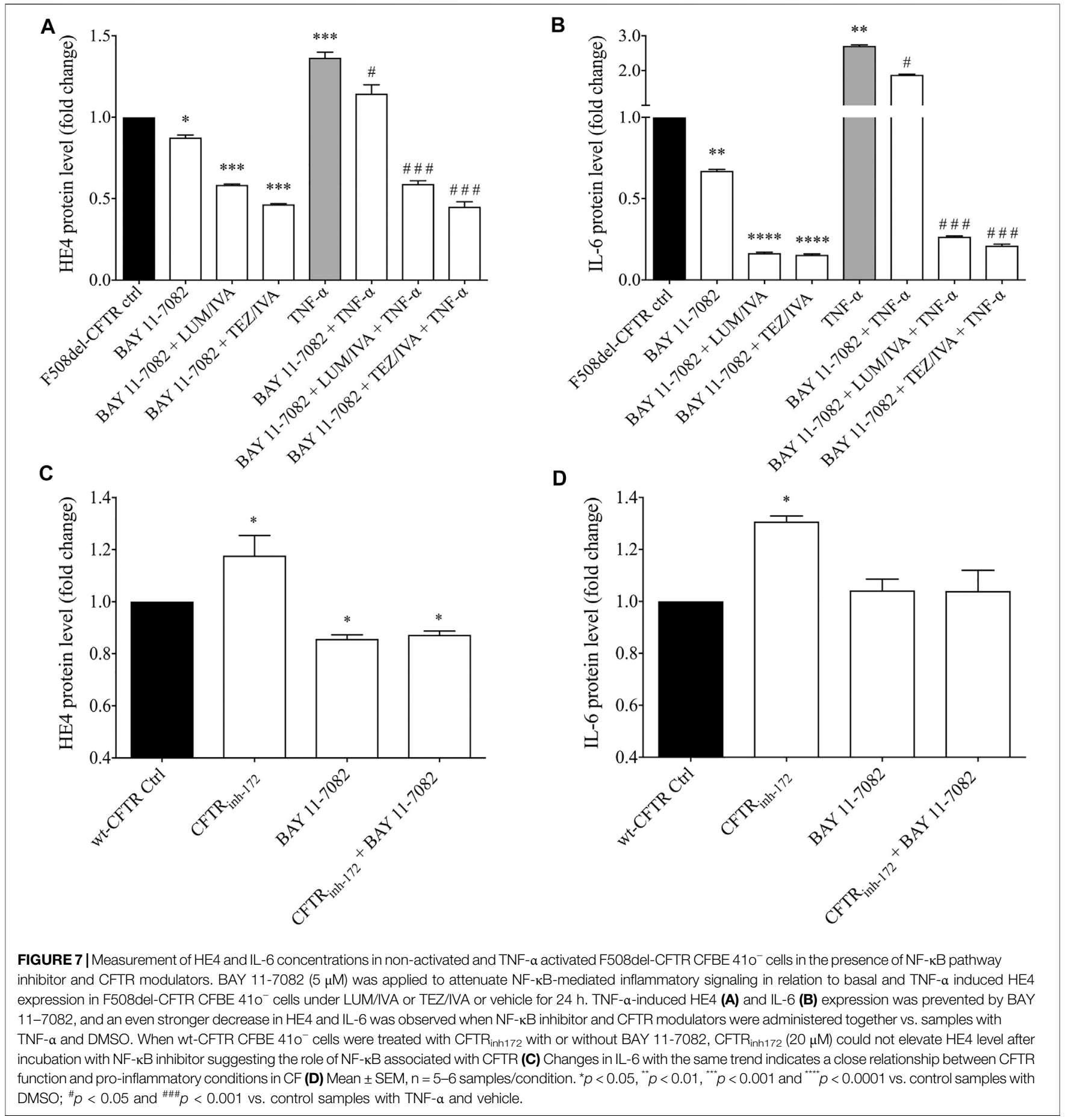

level due to the activation of residual CFTR function of bronchial epithelial cells with F508del-CFTR. However, this particular effect was lower than what we observed in wt-CFTR cells. Finally, when FSK/IBMX was used in combination with CFTR modulators in F508del-CFTR CFBE $410^{-}$cells, HE4 further decreased, especially with TEZ/IVA vs. control samples with DMSO. All these HE4 results are consistent with the change in $\mathrm{Cl}^{-}$current under different treatments (Figure 1A). Based on our results, impaired CFTR function increases baseline HE4 expression in CF airway epithelial cells, which in turn could be efficiently lowered by CFTR modulators.

Next, we wanted to study if WFDC2/HE4 expression could be upregulated in F508del-CFTR CFBE $410^{-}$cells cultures, so these cells were stimulated in vitro with TNF- $\alpha$ for short and long time periods (i.e., for 1 up to $168 \mathrm{~h}$ ). HE4 mRNA level increased following just $1 \mathrm{~h}$ of treatment and remained high for up to $4 \mathrm{~h}$ but returned to the baseline by $24 \mathrm{~h}$. Afterward, when HE4 expression was determined up to $168 \mathrm{~h}$, an even higher 
expression of HE4 was observed in TNF- $\alpha$ stimulated CFBE $410^{-}$ cells. To monitor the pro-inflammatory effect of TNF- $\alpha$ on these CFBE cells upon HE4 overexpression, IL6, IL8, and IL1B mRNAs were also analyzed by RT-qPCR in a similar manner. Comparable patterns were found in IL-6 and IL-8 levels as they were upregulated and changed throughout the experiment, while elevated IL1B expression varied only moderately at the different time points. These results indicate that not only proinflammatory cytokines are upregulated in CF (Nixon et al., 1998; Cantin et al., 2015), but the expression of HE4 can also be triggered under inflammatory circumstances. As a result, HE4 protein level was significantly elevated after $4 \mathrm{~h}$ and gradually increased up to 1 week of treatment, while IL-6 protein concentration was significantly augmented at all time points. Our results are thus reminiscent of a previous report on similar time-dependent regulation of HE4 expression via TLR2 mediated pathway including NF- $\kappa \mathrm{B}$ signaling in cancer cells (Janeckova et al., 2015). Similarly, SLPI and Elafin were also induced in response to TNF- $\alpha$ in human alveolar epithelial cells (Sallenave et al., 1994).

Chronic inflammation via upregulated NF- $\mathrm{B}$ signaling leads to gradual progress of structural damage of airways in CF (Cantin et al., 2015). Based on recent findings, ionic imbalance also generated NLRP3-inflammasome activation in CFBE cells exaggerated by LPS/ATP that caused increased IL-18 secretion (Lara-Reyna et al., 2020). To investigate the correlation between enhanced NF- $\kappa B$ pathway caused by CFTR dysfunction and abnormal HE4 expression in CFBE cells, the activity of NF- $\kappa \mathrm{B}$ pathway via p65 nuclear translocation was visualized by fluorescence microscopy in wt-CFTR and F508del-CFTR CFBE $410^{-}$cells with or without TNF- $a$ treatment. There was a significantly higher basal level of p65 nuclear positivity in F508del-CFTR vs. wt-CFTR CFBE $410^{-}$cells that was in accordance with former results (Wang et al., 2016). These authors also reported that wt-CFTR regulated TNF- $\alpha$ signaling by enhancing TRADD degradation. By reducing the levels of TRADD, wt-CFTR suppressed downstream the proinflammatory NF- $\kappa B$ signaling, while suppression of NF- $\kappa B$ activation failed in CF cells expressing F508del-CFTR (Wang et al., 2016). When our CFBE $410^{-}$cells cultures were activated with TNF- $\alpha$, the difference in p65 nuclear translocation between normal and deficient CFBE cells was more pronounced. These findings imply that enhanced baseline and triggered NF- $\kappa \mathrm{B}$ signaling and abnormal HE4 expression in vitro could be related to each other. Therefore, we raised the question of whether application of LUM/IVA or TEZ/IVA treatment could reduce basal and TNF- $\alpha$ induced NF- $\kappa \mathrm{B}$ signaling that is responsible for lower HE4 concentrations in the supernatant. We found that CFTR modulators not only attenuated $\mathrm{p} 65$ positivity in unstimulated F508del-CFTR CFBE $410^{-}$cells, but a significant reduction was also observed in TNF- $\alpha$ after treatment with CFTR modulators. Decreased baseline and induced IL-6 protein levels in the supernatants of these cells suggest that there are additional anti-inflammatory properties of CFTR modulators apart from decreasing HE4 expression as recently evidenced by others (Ruffin et al., 2018). Overall, these data indicate that the inflammatory status of CF airway epithelial cells could be positively mitigated by CFTR modulators. Our observation is supported by data regarding reduced NLRP3-inflammasome activation in PBMCs observed in CF subjects receiving LUM/ IVA or TEZ/IVA via decreased Caspase- 1 activity by 3 months of treatment (Jarosz-Griffiths et al., 2020) and by the facts that treatment with Orkambi ${ }^{\circledR}$ restored CFTR dependent chloride efflux (Favia et al., 2020), decreased IL-18 and TNF- $\alpha$ expression in PBMCs (Jarosz-Griffiths et al., 2020), and improved airway epithelial repair (Adam et al., 2018), while Symdeko ${ }^{\circledR}$ downregulated serum IL-1 $\beta$ level in CF subjects (Jarosz-Griffiths et al., 2020). Intriguingly, there were available data, which are contradictory to our results on reduced baseline and TNF- $\alpha$ stimulated IL- 6 concentrations in response to LUM/ IVA or TEZ/IVA in F508del-CFTR CFBE $410^{-}$cells. Stanton et al. reported that PAO1 (MIM:615,854) alone induced a substantial expression of IL-6 and IL-8, while stimulated F508del-CFTR $\mathrm{Cl}^{-}$secretion was reduced despite co-treatment with LUM or LUM/IVA, and IL-6 and IL-8 levels remained unaffected (Stanton et al., 2015). Recently, Laselva et al. demonstrated that the levels of IL-6, IL- 8 and TNF- $\alpha$ were reduced following Orkambi ${ }^{\circledR}$ and rescued F508del-CFTR HBE cells, which were exposed to PAO1, but only in the presence of antimicrobial peptide or tobramycin (Laselva et al., 2020). In both previous studies, $\mathrm{PAO} 1$ resulted in a significant decrease in CFTR expression, but Orkambi ${ }^{\circledR}$ administration was not able to "compensate" for the pro-inflammatory effect of PAO1, which could reflect differences between PAO1 and TNF- $\alpha$ pathway. In our experiments, TNF- $\alpha$ as a pro-inflammatory mediator enhanced IL- 6 levels via the NF- $\kappa$ B similar to impaired CFTR function that was prevented by the preincubation with CFTR modulators (Figure 5C) with or without inhibitor BAY 117082 (Figure 7B). Based on the immunofluorescence analysis of CFBE $410^{-}$cells for p65 positivity, either LUM/IVA or TEZ/IVA reduced p65 nuclear translocation leading to lower IL-6 and HE4 levels. Thus, despite this discrepancy, we are convinced that our in vitro data support the anti-inflammatory properties of CFTR modulators.

Apart from baseline levels of HE4, TNF- $\alpha$ induced HE4 levels also declined with applied CFTR modulators in F508del-CFTR CFBE $410^{-}$cells. Furthermore, TNF- $\alpha$ stimulated CFBE $410^{-}$ cells with wt-CFTR demonstrated lowered HE4 levels in the presence of CFTR activator FSK/IBMX. Since we found enhanced level of NF- $\kappa B$ pathway activity in CFBE cells bearing the F508del-CFTR in contrast to wt-CFTR cells at both basal level and upon TNF- $\alpha$ activation, we finally investigated whether $\mathrm{HE} 4$ expression could be altered using specific NF- $\kappa B$ pathway inhibitors. For this purpose, the commercially available BAY 11-7082 inhibitor was applied in vitro to attenuate $\mathrm{NF}-\kappa \mathrm{B}$ mediated signaling in deficient CFBE cells in the absence or presence of TNF- $\alpha$ pretreatment and CFTR modulators. Under these experimental conditions we then determined supernatant HE4 and IL-6 protein levels. We found that both inhibitors significantly lowered the baseline and induced protein level of HE4 and IL-6 (Figures 7A,B). Our data implicate that CFTR mediated NF- $\kappa B$ pathway is involved in the regulation of HE4 expression in CFBE cells. The rescue of CFTR function in either unstimulated or TNF- $\alpha$-activated F508del- 

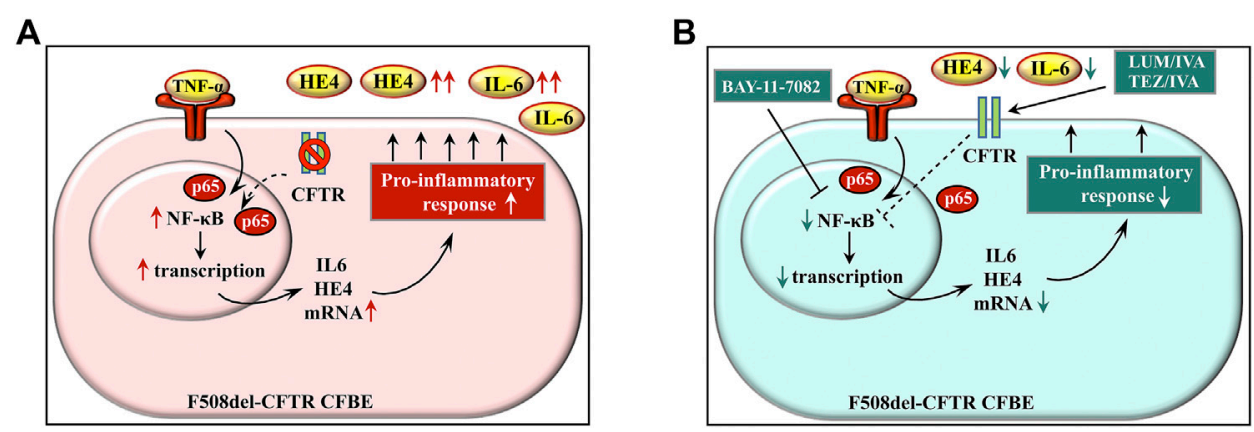

FIGURE 8 | Schematic figure about the model on the regulation of HE4 expression in CF via CFTR and pro-inflammatory signaling. There is an increased basal expression of HE4 with cytokines (e.g., IL-6) in lung epithelial cells of CF that is caused by CFTR dysfunction and external pro-inflammatory stimuli, e.g., TNF-a via the NFкB pathway (A) Blocking the effect of TNF-a by BAY 11-7082 or using CFTR modulators (LUM/IVA or TEZ/IVA) can effectively reduce pro-inflammatory response in CFBE cells by suppressing the NF-kB pathway with a still unknown mechanism (dotted line) that prevents IL-6 and HE4 overexpression (B) Abbreviations: CFBE cell: cystic fibrosis bronchial epithelial cell, CFTR: cystic fibrosis transmembrane conductance regulator, TNF-a: tumor necrosis factor alpha, HE4: human epididymis protein 4, IL-6: interleukin-6, NF-kB: nuclear factor-kappa B.

CFTR CFBE $410^{-}$cells consistently documented reduced HE4 levels not only in the current in vitro samples (Figures 2A, 6A), but also in CF patients who were on CFTR modulator therapy (Figure 2C). These findings were supported with reduced HE4 supernatant levels following BAY 11-7082 CFBE cell culture treatment within the same conditions. In addition, we previously found that in those CF subjects who suffered from more severe inflammation (with acute exacerbation) showing higher CRP values, serum HE4 concentrations were also much higher (Nagy et al., 2016).

Overall, we propose that dual regulation by CFTR and proinflammatory signaling via the NF- $\kappa \mathrm{B}$ pathway upregulates HE4 expression in CFBE. Bitam and her colleagues formerly reported significantly enhanced CFTR maturation and related chloride currents in F508del-CFTR transfected HeLa cells and primary bronchial epithelial cell cultures after $6 \mathrm{~h}$ of treatment with TNFa, however, CFTR function was only slightly improved within the period of $24 \mathrm{~h}$ (Bitam et al., 2015). Therefore, even if TNF- $\alpha$ might rescue CFTR to a certain extent by $24 \mathrm{~h}$ under our conditions, HE4 expression was not sufficiently influenced. Earlier, cytokine-mediated induction of Elafin in pulmonary epithelial cells was described to be regulated via an NF- $\kappa B$ site within the proximal promoter of Elafin (Bingle et al., 2001). In addition, a potential NF- $\kappa \mathrm{B}$ binding site can be indicated at position -322 relative to the HE4 promoter region (Chen et al., 1998). Hence, we suppose that HE4 is also transcriptionally regulated by the NF- $\kappa \mathrm{B}$, however, further e.g. ChIP-seq-based expression studies are required to support the assertion of any functional relationship.

The manuscript has limitations. Firstly, we have not analyzed whether the absence of CFTR function or the presence of misfolded F508del-CFTR was the cause of increased HE4 expression. Secondly, CF patients on TEZ/IVA medication could not be enrolled for plasma HE4 measurement into this study, because such samples are currently not available, either from CFFT nor from our national studies. Thirdly, the mechanistic relationship between the CF-related inflammation and the expression of HE4 was not fully investigated, thus additional studies are required to reveal further related pathogenetic aspects.

In conclusion, we provide evidence that there is a direct relationship between CFTR function and pro-inflammatory response in F508del-CFTR CFBE cells in terms of increased expression of HE4 measured by its concentrations in the cell culture supernatant in vitro (Figure 8). CFTR corrector and potentiator administration restores inflammatory balance via correcting CFTR function and reducing NF- $\kappa \mathrm{B}$ p65 nuclear translocation in CFBE cells as reflected by lower $\mathrm{HE} 4$ concentrations in vitro and in vivo. Finally, we believe that our study opens novel research avenues in terms of providing evidence for the diagnostic utility of plasma biomarker HE4 in terms of its use in efficient monitoring of anti-inflammatory properties of CFTR modulators.

\section{DATA AVAILABILITY STATEMENT}

The raw data supporting the conclusions of this article will be made available by the authors, without undue reservation, to any qualified researcher.

\section{ETHICS STATEMENT}

The studies involving human participants were reviewed and approved by Regional Ethics Committee of the University of Debrecen (permit number: 4813-2017). Written informed consent to participate in this study was provided by the participants' legal guardian/next of kin.

\section{AUTHOR CONTRIBUTIONS}

$\mathrm{BN}$ designed and performed experiments, analyzed data, provided overall direction and wrote the manuscript. $\mathrm{ZB}, \mathrm{ZF}$, TS, FF, and JV performed experiments; LC and GP analyzed data; 
MM, GP, MA, and IB revised the manuscript and provided critical revisions of the intellectual content of this manuscript. All authors edited, reviewed, and approved the final version of the manuscript.

\section{FUNDING}

This research was funded by an OTKA grant (FK 135327) to $\mathrm{BN}$ and the GINOP-2.3.2-15-2016-00043 project. The project is co-financed by the European Union and the European Regional Development Fund. The study was also supported by grants from the Ministry of Health $00064203 / 6003$ and the Ministry of Youth Education and Sports (Both Czechia) LM2018132 to MM. Work in MDA lab is supported by UIDB/04046/2020 and UIDP/04046/2020 centre grants both from FCT/MCTES Portugal (to BioISI) and research grant (to MDA) SRC 013 from CF Trust-UK. ZF is supported by the

\section{REFERENCES}

Adam, D., Bilodeau, C., Sognigbé, L., Maillé, É., Ruffin, M., and Brochiero, E. (2018). CFTR Rescue with VX-809 and VX-770 Favors the Repair of Primary Airway Epithelial Cell Cultures from Patients with Class II Mutations in the Presence of Pseudomonas aeruginosa Exoproducts. J. Cystic Fibrosis 17, 705-714. doi:10.1016/j.jcf.2018.03.010

Barnaby, R., Koeppen, K., Nymon, A., Hampton, T. H., Berwin, B., Ashare, A., et al. (2018). Lumacaftor (VX-809) Restores the Ability of CF Macrophages to Phagocytose and Kill Pseudomonas aeruginosa. Am. J. Physiol.-Lung Cell Mol. Physiol. 314, L432-L438. doi:10.1152/ajplung.00461.2017

Bene, Z., Fejes, Z., Macek, M., Jr., Amaral, M. D., Balogh, I., and Nagy, B., Jr. (2020). Laboratory Biomarkers for Lung Disease Severity and Progression in Cystic Fibrosis. Clinica Chim. Acta 508, 277-286. doi:10.1016/j.cca.2020.05.015

Billet, A., Froux, L., Hanrahan, J. W., and Becq, F. (2017). Development of Automated Patch Clamp Technique to Investigate CFTR Chloride Channel Function. Front. Pharmacol. 8, 195. doi:10.3389/fphar.2017.00195

Bingle, L., Cross, S. S., High, A. S., Wallace, W. A., Rassl, D., Yuan, G., et al. (2006). WFDC2 (HE4): a Potential Role in the Innate Immunity of the Oral Cavity and Respiratory Tract and the Development of Adenocarcinomas of the Lung. Respir. Res. 7, 61. doi:10.1186/1465-9921-7-61

Bingle, L., Tetley, T. D., and Bingle, C. D. (2001). Cytokine-Mediated Induction of the Human Elafin Gene in Pulmonary Epithelial Cells Is Regulated by Nuclear Factor- к B. Am. J. Respir. Cel. Mol. Biol. 25, 84-91. doi:10.1165/ajrcmb.25.1. 4341

Bitam, S., Pranke, I., Hollenhorst, M., Servel, N., Moquereau, C., Tondelier, D., et al. (2015). An Unexpected Effect of TNF- $\alpha$ on F508del-CFTR Maturation and Function. F1000Res 4, 218. doi:10.12688/f1000research.6683.1

Boinot, C., Jollivet Souchet, M., Ferru-Clément, R., and Becq, F. (2014). Searching for Combinations of Small-Molecule Correctors to Restore F508del-Cystic Fibrosis Transmembrane Conductance Regulator Function and Processing. J. Pharmacol. Exp. Ther. 350, 624-634. doi:10.1124/jpet.114.214890

Bonfield, T. L., Hodges, C. A., Cotton, C. U., and Drumm, M. L. (2012). Absence of the Cystic Fibrosis Transmembrane Regulator (Cftr) from Myeloid-Derived Cells Slows Resolution of Inflammation and Infection. J. Leukoc. Biol. 92, 1111-1122. doi:10.1189/jlb.0412188

Cantin, A. M., Hartl, D., Konstan, M. W., and Chmiel, J. F. (2015). Inflammation in Cystic Fibrosis Lung Disease: Pathogenesis and Therapy. J. Cystic Fibrosis 14, 419-430. doi:10.1016/j.jcf.2015.03.003

Chen, F. E., Huang, D.-B., Chen, Y.-Q., and Ghosh, G. (1998). Crystal Structure of P50/p65 Heterodimer of Transcription Factor NF-Kb Bound to DNA. Nature 391, 410-413. doi:10.1038/34956

Clarke, L. A., Sousa, L., Barreto, C., and Amaral, M. D. (2013). Changes in Transcriptome of Native Nasal Epithelium Expressing F508del-CFTR and
UNKP-20-4-II-DE-197 New National Excellence Program of The Ministry for Innovation and Technology.

\section{ACKNOWLEDGMENTS}

The authors thank Ms Edit Kalina, BSc for excellent technical assistance. BN was a recipient of the Lajos Szodoray Grant (Faculty of Medicine, University of Debrecen). CFFT (Bethesda, MD, United States) is acknowledged to supply the human samples to this study.

\section{SUPPLEMENTARY MATERIAL}

The Supplementary Material for this article can be found online at: https://www.frontiersin.org/articles/10.3389/fphar.2021.592184/ full\#supplementary-material

Intersecting Data from Comparable Studies. Respir. Res. 14, 38. doi:10.1186/ 1465-9921-14-38

De Boeck, K., and Amaral, M. D. (2016). Progress in Therapies for Cystic Fibrosis. Lancet Respir. Med. 4, 662-674. doi:10.1016/s2213-2600(16)00023-0

Eidelman, O., Srivastava, M., Zhang, J., Leighton, X., Murtie, J., Jozwik, C., et al. (2001). Control of the Proinflammatory State in Cystic Fibrosis Lung Epithelial Cells by Genes from the TNF-Ar/nfkb Pathway. Mol. Med. 7, 523-534. doi:10. 1007/bf03401858

Favia, M., Gallo, C., Guerra, L., De Venuto, D., Diana, A., Polizzi, A. M., et al. (2020). Treatment of Cystic Fibrosis Patients Homozygous for F508del with Lumacaftor-Ivacaftor (Orkambi) Restores Defective CFTR Channel Function in Circulating Mononuclear Cells. Int. J. Mol. Sci. 21, 2398. doi:10.3390/ ijms 21072398

Fejes, Z., Czimmerer, Z., Szük, T., Póliska, S., Horváth, A., Balogh, E., et al. (2018). Endothelial Cell Activation Is Attenuated by Everolimus via Transcriptional and Post-transcriptional Regulatory Mechanisms after Drug-Eluting Coronary Stenting. PLoS One 13, e0197890. doi:10.1371/ journal.pone.0197890

Hisert, K. B., Heltshe, S. L., Pope, C., Jorth, P., Wu, X., Edwards, R. M., et al. (2017). Restoring Cystic Fibrosis Transmembrane Conductance Regulator Function Reduces Airway Bacteria and Inflammation in People with Cystic Fibrosis and Chronic Lung Infections. Am. J. Respir. Crit. Care Med. 195, 1617-1628. doi:10. 1164/rccm.201609-1954oc

Hunter, M. J., Treharne, K. J., Winter, A. K., Cassidy, D. M., Land, S., and Mehta, A. (2010). Expression of Wild-type CFTR Suppresses NF-kappaB-Driven Inflammatory Signalling. PLoS One 5, e11598. doi:10.1371/journal.pone. 0011598

Janeckova, L., Pospichalova, V., Fafilek, B., Vojtechova, M., Tureckova, J., Dobes, J., et al. (2015). HIC1 Tumor Suppressor Loss Potentiates TLR2/NF-Kb Signaling and Promotes Tissue Damage-Associated Tumorigenesis. Mol. Cancer Res. 13, 1139-1148. doi:10.1158/1541-7786.mcr-15-0033

Jarosz-Griffiths, H. H., Scambler, T., Wong, C. H., Lara-Reyna, S., Holbrook, J., Martinon, F., et al. (2020). Different CFTR Modulator Combinations Downregulate Inflammation Differently in Cystic Fibrosis. Elife 9, e54556. doi:10.7554/elife.54556

Kmit, A., Marson, F. A. L., Pereira, S. V.-N., Vinagre, A. M., Leite, G. S., Servidoni, M. F., et al. (2019). Extent of rescue of F508del-CFTR function by VX-809 and VX-770 in human nasal epithelial cells correlates with SNP rs7512462 in SLC26A9 gene in F508del/F508del Cystic Fibrosis patients. Biochim. Biophys. Acta (Bba) - Mol. Basis Dis. 1865, 1323-1331. doi:10.1016/j.bbadis. 2019.01.029

Lara-Reyna, S., Holbrook, J., Jarosz-Griffiths, H. H., Peckham, D., and McDermott, M. F. (2020). Dysregulated Signalling Pathways in Innate Immune Cells with Cystic Fibrosis Mutations. Cell. Mol. Life Sci. 77, 4485-4503. In press. doi:10. 1007/s00018-020-03540-9 
Laselva, O., Stone, T. A., Bear, C. E., and Deber, C. M. (2020). Anti-Infectives Restore ORKAMBI Rescue of F508del-CFTR Function in Human Bronchial Epithelial Cells Infected with Clinical Strains of P. aeruginosa. Biomolecules 10, 334. doi:10.3390/biom10020334

Nagy, B., Jr., Bene, Z., Fejes, Z., Heltshe, S. L., Reid, D., Ronan, N. J., et al. (2019). Human Epididymis Protein 4 (HE4) Levels Inversely Correlate with Lung Function Improvement (Delta FEV1) in Cystic Fibrosis Patients Receiving Ivacaftor Treatment. J. Cystic Fibrosis 18, 271-277. doi:10.1016/j.jcf.2018. 08.013

Nagy, B., Jr., Nagy, B., Fila, L., Clarke, L. A., Gönczy, F., Bede, O., et al. (2016). Human Epididymis Protein 4: A Novel Serum Inflammatory Biomarker in Cystic Fibrosis. Chest 150, 661-672. doi:10.1016/j.chest.2016.04.006

Nixon, L. S., Yung, B., Bell, S. C., Stuart Elborn, J., and Shale, D. J. (1998). Circulating Immunoreactive Interleukin-6 in Cystic Fibrosis. Am. J. Respir. Crit. Care Med. 157, 1764-1769. doi:10.1164/ajrccm.157.6.9704086

Pranke, I. M., Hatton, A., Simonin, J., Jais, J. P., Le Pimpec-Barthes, F., Carsin, A., et al. (2017). Correction of CFTR Function in Nasal Epithelial Cells from Cystic Fibrosis Patients Predicts Improvement of Respiratory Function by CFTR Modulators. Sci. Rep. 7, 7375. doi:10.1038/s41598-017-07504-1

Ramsey, B. W., Davies, J., McElvaney, N. G., Tullis, E., Bell, S. C., Dřevínek, P., et al. (2011). A CFTR Potentiator in Patients with Cystic Fibrosis and theG551DMutation. N. Engl. J. Med. 365, 1663-1672. doi:10.1056/ nejmoal105185

Rowe, S. M., Heltshe, S. L., Gonska, T., Donaldson, S. H., Borowitz, D., Gelfond, D., et al. (2014). GOAL Investigators of the Cystic Fibrosis Foundation Therapeutics Development Network. (Clinical Mechanism of the Cystic Fibrosis Transmembrane Conductance Regulator Potentiator Ivacaftor in G551D-Mediated Cystic Fibrosis. Am. J. Respir. Crit. Care Med. 190, 175-184. doi:10.1164/rccm.201404-0703oc

Rowe, S. M., Miller, S., and Sorscher, E. J. (2005). Cystic Fibrosis. N. Engl. J. Med. 352, 1992-2001. doi:10.1056/nejmra043184

Ruffin, M., Roussel, L., Maillé, É., Rousseau, S., and Brochiero, E. (2018). Vx-809/Vx-770 Treatment Reduces Inflammatory Response to Pseudomonas aeruginosa in Primary Differentiated Cystic Fibrosis Bronchial Epithelial Cells. Am. J. Physiol.-Lung Cell Mol. Physiol. 314, L635-L641. doi:10.1152/ajplung.00198.2017

Sallenave, J. M., Shulmann, J., Crossley, J., Jordana, M., and Gauldie, J. (1994). Regulation of Secretory Leukocyte Proteinase Inhibitor (SLPI) and Elastase- specific Inhibitor (ESI/elafin) in Human Airway Epithelial Cells by Cytokines and Neutrophilic Enzymes. Am. J. Respir. Cell Mol. Biol. 11, 733-741. doi:10. 1165/ajrcmb.11.6.7946401

Small, D. M., Doherty, D. F., Dougan, C. M., Weldon, S., and Taggart, C. C. (2017). The Role of Whey Acidic Protein Four-Disulfide-Core Proteins in Respiratory Health and Disease. Biol. Chem. 398, 425-440. doi:10.1515/hsz-2016-0262

Stanton, B. A., Coutermarsh, B., Barnaby, R., and Hogan, D. (2015). Pseudomonas aeruginosa Reduces VX-809 Stimulated F508del-CFTR Chloride Secretion by Airway Epithelial Cells. PLoS One 10, e0127742. doi:10.1371/journal.pone. 0127742

Taylor-Cousar, J. L., Munck, A., McKone, E. F., van der Ent, C. K., Moeller, A., Simard, C., et al. (2017). Tezacaftor-Ivacaftor in Patients with Cystic Fibrosis Homozygous for Phe508del. N. Engl. J. Med. 377, 2013-2023. doi:10.1056/ nejmoa1709846

Vij, N., Mazur, S., and Zeitlin, P. L. (2009). CFTR Is a Negative Regulator of NFkappaB Mediated Innate Immune Response. PLoS One 4, e4664. doi:10. 1371/journal.pone.0004664

Wainwright, C. E., Elborn, J. S., Ramsey, B. W., Marigowda, G., Huang, X., Cipolli, M., et al. (2015). TRAFFIC Study Group; TRANSPORT Study GroupLumacaftor-Ivacaftor in Patients with Cystic Fibrosis Homozygous for Phe508del CFTR. N. Engl. J. Med. 373, 220-231. doi:10.1056/nejmoa1409547

Wang, H., Cebotaru, L., Lee, H. W., Yang, Q., Pollard, B. S., Pollard, H. B., et al. (2016). CFTR Controls the Activity of NF-Kb by Enhancing the Degradation of TRADD. Cell. Physiol. Biochem. 40, 1063-1078. doi:10. $1159 / 000453162$

Conflict of Interest: The authors declare that the research was conducted in the absence of any commercial or financial relationships that could be construed as a potential conflict of interest.

Copyright (C) 2021 Bene, Fejes, Szanto, Fenyvesi, Váradi, Clarke, Panyi, Macek, Amaral, Balogh and Nagy. This is an open-access article distributed under the terms of the Creative Commons Attribution License (CC BY). The use, distribution or reproduction in other forums is permitted, provided the original author(s) and the copyright owner(s) are credited and that the original publication in this journal is cited, in accordance with accepted academic practice. No use, distribution or reproduction is permitted which does not comply with these terms. 